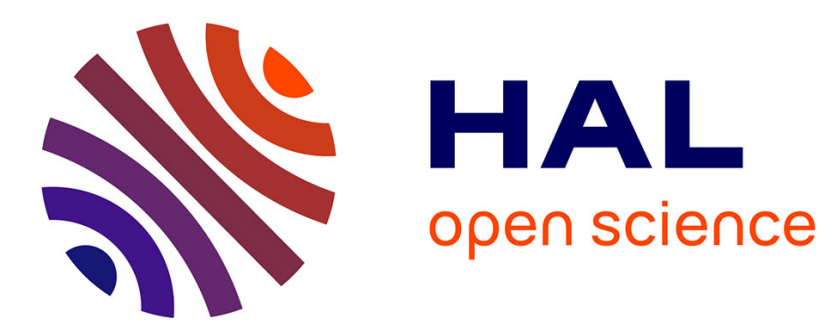

\title{
A Poromechanical Model for Sorption Hysteresis in Nanoporous Polymers
}

Mingyang Chen, Benoit Coasne, Robert Guyer, Dominique Derome, Jan

Carmeliet

\section{- To cite this version:}

Mingyang Chen, Benoit Coasne, Robert Guyer, Dominique Derome, Jan Carmeliet. A Poromechanical Model for Sorption Hysteresis in Nanoporous Polymers. Journal of Physical Chemistry B, 2020, 124 (39), pp.8690-8703. 10.1021/acs.jpcb.0c04477 . hal-02989995

\section{HAL Id: hal-02989995 \\ https://hal.science/hal-02989995}

Submitted on 5 Nov 2020

HAL is a multi-disciplinary open access archive for the deposit and dissemination of scientific research documents, whether they are published or not. The documents may come from teaching and research institutions in France or abroad, or from public or private research centers.
L'archive ouverte pluridisciplinaire HAL, est destinée au dépôt et à la diffusion de documents scientifiques de niveau recherche, publiés ou non, émanant des établissements d'enseignement et de recherche français ou étrangers, des laboratoires publics ou privés. 


\title{
A Poromechanical Model for Sorption Hysteresis in Nanoporous Polymers
}

\author{
Mingyang Chen ${ }^{1, *}$, Benoit Coasne ${ }^{2}$, Robert Guyer ${ }^{3}$, Dominique Derome $^{4}$, Jan Carmeliet ${ }^{1}$ \\ 1 Chair of Building Physics, Department of Mechanical and Process Engineering, ETH Zurich, \\ 8093 Zurich, Switzerland \\ 2 Univ. Grenoble Alpes, CNRS, LIPhy, 38000 Grenoble, France \\ 3 Department of Physics, University of Nevada, Reno, NV, 89557, USA \\ 4 Department of Civil and Building Engineering, Université de Sherbrooke, 2500, Sherbrooke, \\ Cananda \\ * Correspondence and requests should be addressed to M. C. (minchen@ethz.ch)
}

\begin{abstract}
Sorption hysteresis in nanoporous polymer is an intriguing phenomenon that involves coupling between sorption and deformation. Based on the mechanism revealed at the microscopic level using molecular simulation, a poromechanical model is developed capturing all relevant physics and yielding a quantitative description. In this model, the coupling between sorption and deformation is described by a poromechanics framework. More in detail, an upscaling process from the molecular mechanism is implemented to model the hysteresis through the state change of each element upon deformation. We provide two solutions of the model, a numerical one based on the finite element method and an analytical one based on uniform strain assumption. The results from both solutions agree well with the molecular simulation and experimental results, therefore capturing and describing adequately sorption hysteresis. The developed model illustrates that water forms different structural distributions upon adsorption and desorption. A parametric study shows that sorption hysteresis is influenced by material properties. We find that a softer material with stronger adsorbent-adsorbate interaction tends to exhibit more profound sorption hysteresis. The developed model, which relies on the concepts of sorption-deformation coupling and multi-scale modeling from atomistic simulations to domain dependent theory, paves the way for a new direction of modeling sorption hysteresis.
\end{abstract}




\section{Introduction}

Sorption hysteresis has been observed in various adsorbent-adsorbate systems with sorption loops in ad- and desorption of different shapes and sizes. ${ }^{1-5}$ Depending on the material and pore structure, the origins and features of sorption hysteresis can be very different. For nanoporous polymers, an important feature of the hysteresis is its persistence over the entire hygroscopic range, i.e. $0 \%-100 \%$ relative humidity $(\mathrm{RH}){ }^{6-10}$. Such a feature is not present in the typical hysteresis types defined by the International Union of Pure and Applied Chemistry (IUPAC) ${ }^{11}$, where hysteresis manifests itself only at higher relative vapor pressure and is mainly attributed to capillary condensation hysteresis in mesoporous materials. This persisting hysteresis has been observed for nearly a century in nanoporous polymers and related materials, such as wood and other materials of vegetal origin, and remains a perplexing - only partly explained - feature ${ }^{8,9}$.

There are some hypotheses venturing at explaining the origin of hysteresis in nanoporous polymers. Capillary condensation hysteresis and pore ink-bottle effects ${ }^{6}$ are the most common explanations for sorption hysteresis. ${ }^{12,13}$ However, attributing the hysteresis to the non-uniqueness of the surfaces of capillary menisci of liquid water in larger pores cannot apply to nanoporous polymers. The pore sizes of polymers are generally smaller than $2 \mathrm{~nm}$ and capillary condensation/evaporation cannot happen in such small pores. ${ }^{14-17}$ Moreover, in the picture of capillary condensation, low RH sorption consists of the reversible formation of an adsorbed - a feature which is not observed in nanoporous polymers, where hysteresis persists almost over the entire isotherm. Bažant and Bazant 18,19 explain hysteresis, in general and not specifically for nanoporous polymers, as follows. They consider distances between atoms to be non-unique even at the same energy state which means that, at the same chemical potential, two states - of positive or negative pressure - are possible and finally leads to hysteresis. This mechanism is derived under the assumption of rigid sorbent, which means that the hysteresis should also manifest itself when the deformation is constrained. Molecular simulations show that hysteresis vanishes at fixed displacement boundary conditions prohibiting any deformation during sorption for nanoporous polymers ${ }^{14}$, which indicates that this explanation may not apply. The other category of explanations relies on structural changes of nanoporous polymers upon sorption. Urquhart et al. attribute the hysteresis in the sorption of water by cellulose to an irreversible coupling/decoupling process of hydroxyl groups within the cellulose structure. ${ }^{7,10,20}$ The uncoupling occurs during adsorption while the recoupling occurs at a relatively delayed stage in the desorption process, leading to a reduced number of sorption sites in the course of adsorption processes. Along this line, Filby and Maass conclude that there is a greater proportion of bound water in cellulose during desorption than during adsorption. ${ }^{9}$ Though no direct proof was 
given and the mechanism leading to the irreversible coupling and uncoupling of hydroxyl groups was vague, this hypothesis depicted a rough but promising microscopic picture at the molecular level of sorption hysteresis in nanoporous polymers. Chirkova et al. pointed out, when studying wood-related polymers, that hysteresis in wood was caused by the low modulus of elasticity of cell walls, of which pores were closed upon removal of water, blocking the complete release of the water from wood pores. ${ }^{21}$ This explanation related the origin of hysteresis to structural changes of the polymers caused by mechanical deformation. Recently, we verified the hypothesis given above by molecular simulations and proposed a comprehensive explanation of hysteresis based on coupling between sorption and structural change caused by swelling. ${ }^{14}$ By examining the hydrogen bonds, the sorption hysteresis was explained by the fact that polymer swells to form water-polymer hydrogen bonds upon adsorption while these hydrogen bonds do not break upon desorption at the same vapor pressure.

In terms of modeling sorption hysteresis at the mesoscopic scale, the main method is a phenomenological approach named the Independent Domain Theory (IDT). Stemming from the work of Preisach ${ }^{22}$ for magnetism hysteresis, the IDT was developed by Everett for sorption..$^{23,24}$ (See also Ref. ${ }^{25}$ for a revisited version of Everett's IDT and its applications to scanning curves within sorption hysteresis in porous media). IDT decomposes the porous system into an assembly of independent domains. Each domain, which is generally termed as hysteron, has inherent hysteresis, being filled and drained at different vapor pressures independently of its neighbors. The hysteretic feature of whole system can be reproduced by adjusting the population of hysterons, an integrated example is given in Ref. ${ }^{26}$. IDT has a clear physical background when addressing systems with macropores, where the hysteresis at the single pore level is represented by a hysteron. Hysteresis is then the outcome of a set of hysteretic pores described by a distribution of elements each showing a different vapor pressure for filling and emptying. Apart from macroporous media, IDT has also been successfully extended to address the sorption hysteresis in nanoporous materials by implementing sorption features at the nanoscale into the model. This includes for instance works in which the contribution from the liquid film formed prior to capillary condensation was added to the original IDT ${ }^{25}$, Pore network features such as percolation and finite lattice effects were also considered by Pinson et al ${ }^{27}$. In these approaches, the solid hosts are treated as stiff materials so that the impact of deformation on sorption hysteresis is neglected de facto. However, IDT deviates from the key nature of the nanoporous polymers in two aspects. First, no hysteresis is present at single pore level for nanoporous polymers because of the limited pore size, which directly makes the application of the concept of hysteron questionable. Second, the sorption-induced deformation in nanoporous polymers is pronounced and the mechanical interactions between neighboring pores 
are the major cause for the hysteresis according to molecular simulations, whereas the mechanical interaction between neighboring hysterons is omitted in IDT by the assumption of neighbor independence. Though one can still insist on employing IDT to model sorption hysteresis in nanoporous polymers ${ }^{28-31}$, IDT in this case is an unphysical approach, since it does not take into account the mechanical coupling between different elements. Inspired by IDT, a promising option is to include the coupling physics between sorption and deformation leading to mechanical interaction between different elements into the domain theory, extending the framework from 'independent' to 'dependent'. The model with this coupling concept can be referred to as dependent domain model (DDM). Guyer et al. showed that, by implementing the mechanical constitutive relationship together with the sorption-deformation coupling mechanism into the domain theory, one can capture the sorption hysteresis caused by sorption-induced deformation. ${ }^{32}$ The required specification of the coupling constitutive relationships between deformation and sorption can be done based on the poromechanical framework, where the influence of the sorption is integrated by considering an extra stress term - namely the sorption-induced stress. ${ }^{33-38}$ Several extensions have been made in poromechanics to model the coupling of sorption and deformation for nanoporous solids. ${ }^{36,39-43}$ For nanoporous materials with small sorption-induced deformation, the sorptioninduced stress can be calculated from the undeformed configurations assuming negligible deformation impact on the sorption process. For instance, Zhou et al ${ }^{42,44}$ estimated the structural relaxation of wet granular materials using molecular dynamics (MD) including the precalculated sorption-induced stress from density functional theory (DFT). In this approach, by looking at capillary forces at the nanograin level, sorption-induced deformation at the nanoscale such as nonaffine local deformation was discussed in detail. However, for nanoporous polymers with relatively large deformation, like those considered in the present work, the impact of deformation on the sorption process cannot be neglected as the pores of the deformed polymers lead to a new adsorption energy landscape ${ }^{45-47}$. The change of energy landscape can even lead to decrease of sorption amount upon adsorption, which is termed as 'negative gas adsorption'47,48. In practice, such feedback between deformation and adsorption can be modeled by employing hybrid molecular simulation techniques ${ }^{14}$, which account concomitantly for these poromechanical effects. Thus, the combination of DDM and poromechanics serves as a potential candidate to describe sorption hysteresis in nanoporous polymers.

In this work, a theoretical model based on the coupling physics between sorption and deformation as observed at the molecular level is developed by integrating poromechanics with DDM. The constitutive relationship of each domain is built based on poromechanics considering the nonlinear 
constitutive behavior of the material. With the newly developed model, the roles of different parameters regarding mechanical and sorptive properties on hysteresis are studied.

\section{Methods}

\subsection{Mechanism of hysteresis at molecular level}
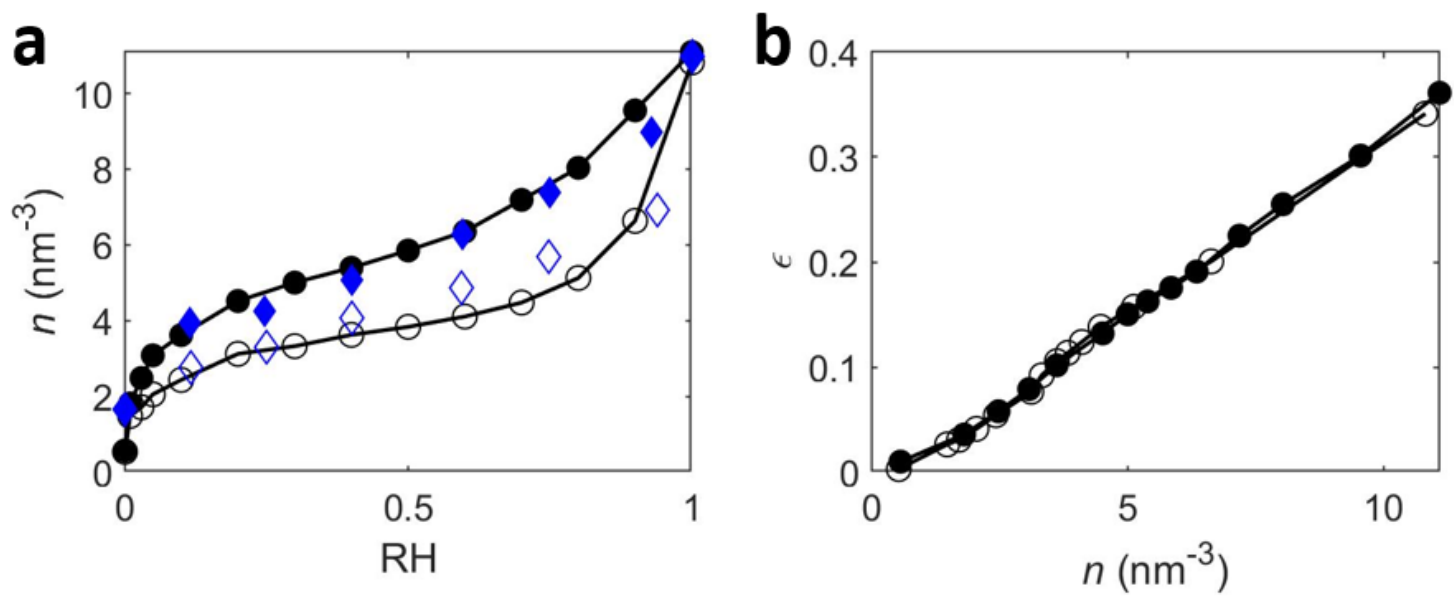

Figure 1 (a) Water sorption isotherm of amorphous cellulose (AC). (b) Strain dependence on sorption amount. Black dots and blue diamond represent the molecular simulations ${ }^{14}$ and experimental results ${ }^{49}$ respectively. Adsorption and desorption are represented by open and closed symbols, respectively.

The DDT proposed in this work for nanoporous polymers is based on the coupling physics found at the molecular scale, which has been documented in Ref. ${ }^{14}$ by a detailed molecular simulation study on amorphous cellulose (AC). Here, for the sake of completeness, we briefly review the mechanism of the coupled phenomenon and related hysteresis. One can refer to Ref. ${ }^{14}$ for details with regard to the molecular simulation. Figure 1(a) shows the water sorption isotherms obtained from molecular simulations and experiments ${ }^{49}$ at $300 \mathrm{~K}$. The sorption amount $n$ is defined as number density of adsorbed water molecules, i.e. $n=N_{\mathrm{W}} / V_{0}$, where $N_{\mathrm{W}}$ is the number of adsorbed water molecule and $V_{0}$ is the volume of dry AC. The sorption amount $n$ increases rapidly with RH in the low RH region, less rapidly mid-course until an inflection point, and then significantly as subsequent sorption occurs. The sorption amount shows a hysteresis over the entire RH range from 0 to 1 . However, when plotted against the volumetric strain $\varepsilon$, which is defined as $\varepsilon=\left(V-V_{0}\right) / V_{0}$, the hysteresis vanishes as shown in Figure 1(b). This indicates that the same amount of water molecules produces the same swelling effect independently of sorption history, which is attributed to sorption-induced swelling being mainly caused by the volume change resulting from adding new water molecules. One should note that this is only valid for homogenized quantities. For local 
deformations and moisture concentrations, this unique relationship may not hold true. Depending on the local pore configuration formed by the polymer chains, the same number of water molecules can lead to different local strains. This can lead to nonaffine local strains and their relationship with the homogenized strain have been discussed in detail by Zhou et $\mathrm{al}^{42}$.

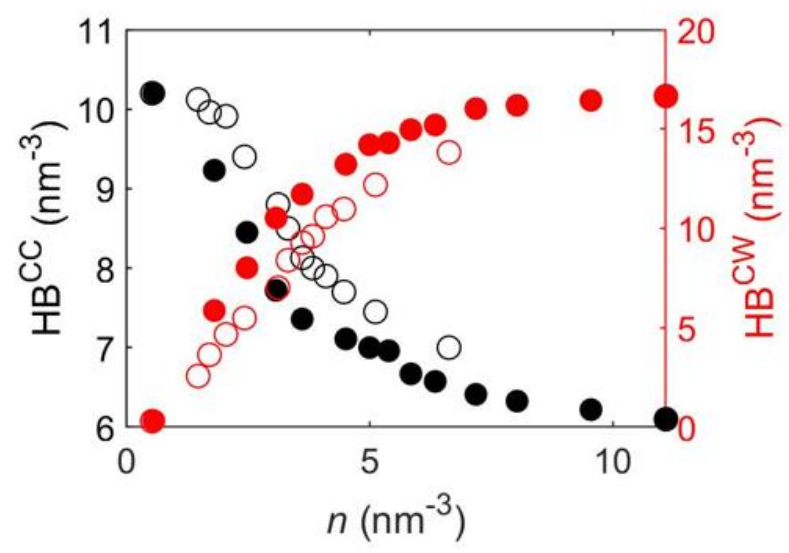

Figure 2 The dependence of cellulose to cellulose ( $\mathrm{HB}^{\mathrm{CC}}$, black) and cellulose to water hydrogen bonds $\left(\mathrm{HB}^{\mathrm{CW}}\right.$, red) on the sorption amount as obtained by means of molecular simulation. Open and closed dots represent adsorption and desorption respectively. Hysteresis is shown between adsorption and desorption of $\mathrm{HB}^{\mathrm{CC}}$ and $\mathrm{HB}^{\mathrm{CW}}$ with $\mathrm{HB}^{\mathrm{CC}}$ lower in desorption and $\mathrm{HB}^{\mathrm{CW}}$ higher in desorption.

Considering the very small pore size in such nanoporous polymers (typically $\sim 1 \mathrm{~nm}$ or less), hysteresis observed here is assumed not to stem from capillary condensation effects ${ }^{50}$. Indeed, there is a large body of theoretical, molecular simulation, and experimental results showing that a simple relationship between the temperature $T$ and the critical pore size $D$ below which pore filling becomes reversible: $T_{\mathrm{c}}-T \sim 4 \lambda T_{\mathrm{c}} / D$ ( $\lambda$ is the size of the confined molecule, $\sim 0.28 \mathrm{~nm}$ for water, and $T_{\mathrm{c}}$ is the bulk critical point) ${ }^{16,51}$. Based on these observations, as the pore size in cellulose is below $\sim 1 \mathrm{~nm}$, no capillary condensation hysteresis is expected at $T=300 \mathrm{~K}$. Using molecular simulation, the sorption hysteresis was found to be related to the formation of a hydrogen bond network as shown in Ref ${ }^{14}$. As shown in Figure 2, more cellulose to cellulose hydrogen bonds, $\mathrm{HB}^{\mathrm{CC}}$, are broken because of swelling when $\mathrm{AC}$ polymer undergoes adsorption. The broken $\mathrm{HB}^{\mathrm{CC}}$ expose hydroxyl groups, which serve as additional sorption sites and are then saturated by newly adsorbed water molecules. As a result, we see the density of cellulose to water hydrogen bonds, $\mathrm{HB}^{\mathrm{CW}}$, increases. However, the $\mathrm{HB}^{\mathrm{CW}}$ in desorption branch is higher than that in the adsorption branch at the same sorption amount, which indicates that the adsorbed water molecules tend to stay with the cellulose chains through $\mathrm{HB}^{\mathrm{CW}}$ and can only be desorbed at very low RH. Physically, this 
is attributed to the high binding energy of $\mathrm{HB}^{\mathrm{CW}} \cdot{ }^{14}$ Consequently, as the broken $\mathrm{HB}^{\mathrm{CC}}$ could not recover before the desorption of water molecules from the hydroxyl groups, we see less $\mathrm{HB}^{\mathrm{CC}}$ in desorption branch. Thus, a hydrogen bond $\mathrm{HB}^{\mathrm{CC}}$ between two polymeric chains is broken at a $\mathrm{RH}$ higher in adsorption than the RH where it reforms in desorption, leading to hysteresis shown in the sorption isotherms.

The aforementioned mechanism is extracted from the microscopic picture at molecular level with the help of molecular simulations of cellulose-water system. Similar sorption hysteresis has also been observed in other nanoporous polymer systems, and the adsorbate is not limited to water ${ }^{52,53}$. Though not strictly verified, the mechanism observed in the cellulose-water system can be generalized and extended to other systems. $\mathrm{As} \mathrm{HB}^{\mathrm{CC}}$ and $\mathrm{HB}^{\mathrm{CW}}$ are specific types of solid-solid and solid-fluid interactions, exchanging them by other types of interactions, such as van-der-Waals forces, does not essentially change the general picture. As a matter of fact, similar mechanism has been proposed by Jeromenok and Weber ${ }^{52}$ to explain the hysteresis of $\mathrm{N}_{2}$ sorption in polymers of intrinsic microporosity (PIMs), where the origin of hysteresis is explained by the opening of restricted-access pores driven by the acting solvation pressure. The microscopic picture depicted by Jeromenok and Weber goes along with $\mathrm{HB}^{\mathrm{CC}}$ breakage due to the swelling effect in the cellulosewater systems.

\subsection{Implementation of molecular mechanism in DDM}

When addressing sorption, a domain theory, either dependent or independent, is formulated on the concept of porous media and generally does not include per se the concept of hydrogen bond. Thus, we develop a new porous medium approach to accommodate with the underlying mechanism of hysteresis. To do so, we model the nanoporous polymeric medium as a collection of domains containing pores following a certain pore size distribution. As shown by the molecular simulation, not all pores are initially accessible to water molecules. For those small pores, the pore size is too small to be accessible for a single water molecule. By defining a critical pore size $R_{\mathrm{C}}$, the pores can be classified into two classes depending on their pore size $R$. Sorption can only happen in pores with a size $R$ larger than $R_{\mathrm{C}}$ so that they are considered accessible or 'open' - this corresponds to a molecular configuration with broken $\mathrm{HB}^{\mathrm{CC}}$ between the chains thus large interchain space. Pores with a size $R$ smaller than $R_{\mathrm{C}}$ are not accessible for water molecules and non-sorptive and are therefore considered 'closed' - this corresponds to a molecular configuration with bonded $\mathrm{HB}^{\mathrm{CC}}$ thus compact interchain space. Upon opening of a pore, the pore will become filled with water molecules, of which the sorption amount increases further with chemical potential and further straining of the pore. With these definitions, we can write: 


$$
n= \begin{cases}n(\mu, \varepsilon) ; & \forall R \geq R_{\mathrm{C}} \\ 0 ; & \forall R<R_{\mathrm{C}}\end{cases}
$$

where $n(\mu, \varepsilon)$ is the sorption amount in open pores dependent on chemical potential and strain. For simplicity, we assume that the sorption isotherm is a function of chemical potential $\mu$ and volumetric strain $\varepsilon$. The detailed form of $n(\mu, \varepsilon)$ will be addressed later.

Consider now two neighboring domains $\mathrm{E}_{1}$ and $\mathrm{E}_{2}$ containing pores of different pore sizes $R_{1}$ and $R_{2}$ as shown in Figure 3(a). Without loss of generality, it is assumed that $R_{1}<R_{2}$. Here we consider the case where $R_{1}<R_{\mathrm{C}}<R_{2}$, which means that initially $\mathrm{E}_{1}$ is non-sorptive and $\mathrm{E}_{2}$ is sorptive. In other words, $E_{1}$ is 'closed' and $E_{2}$ is 'open'. Sorption only happens in $E_{2}$, leading to swelling of element $E_{2}$. Because of the continuity of displacement between the two elements $E_{1}$ and $E_{2}$, element $E_{1}$ will also deform upon the influence of its neighboring element $E_{2}$. Here we consider the free swelling case, i.e., both $E_{1}$ and $E_{2}$ are free to deform. Under such boundary conditions, the stretching effect of the swollen $E_{2}$ leads to a tensile straining of the element $E_{1}$ as well (Figure 3(b)). As a result, the pore size of both elements $\mathrm{E}_{1}$ and $\mathrm{E}_{2}$ increases. In this work, the current pore size $R$ is assumed to have a linear relationship with the volumetric strain, i.e.

$$
R=R_{0}(1+S \varepsilon)
$$

where $R_{0}$ is the initial pore size at zero strain and $S$ is a scaling coefficient connecting volumetric strain and pore size. $\mathrm{E}_{1}$ remains non-sorptive until $R_{1}$ reaches $R_{\mathrm{C}}$, where $\mathrm{E}_{1}$ opens and switches from a non-sorptive pore into a sorptive pore (Figure 3(c,d)). Upon desorption, the adsorbed molecules in $E_{1}$ are difficult to remove because of the strong adsorbent-adsorbate interaction observed in the atomistic results. Thus, water molecules in $\mathrm{E}_{1}$ persists until a much lower $\mathrm{RH}$ compared to the opening RH in adsorption is attained, resulting in hysteresis in both sorption and deformation (Figure 3(e)). 


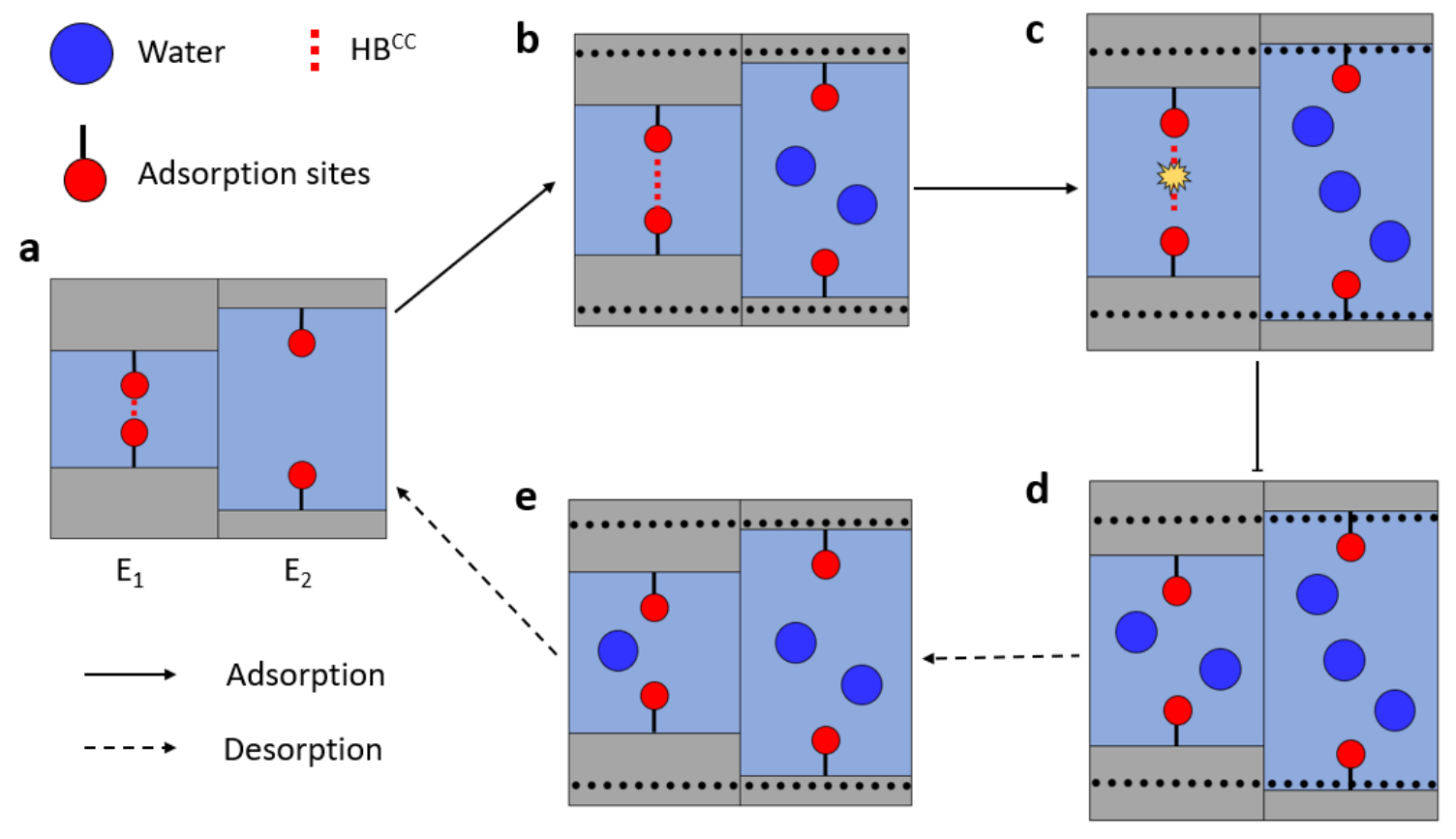

Figure 3 Schematic representation of the hysteresis mechanism implemented in DDM based on the states of two neighboring domains $E_{1}$ and $E_{2}$ : (a) $E_{1}$ and $E_{2}$ are empty at dry state; (b) adsorption happens only in $E_{2}$, resulting in a tensile straining of $E_{1}$ and $E_{2}$; (c) further sorption in $E_{2}$, leading to swelling of the element and breakage of $\mathrm{HB}^{\mathrm{CC}}$ in $\mathrm{E}_{1}$ and switch of $\mathrm{E}_{1}$ from non-sorptive to sorptive as its pore size exceeds $R_{\mathrm{C}}$; (d) increase of sorption amount in $\mathrm{E}_{1}$ after switch; (e) desorption of pores $E_{1}$ and $E_{2}$, showing that $E_{1}$ remains in open state, return to original status only when all water is removed. Horizontal dot lines refer to the original size of the element.

Now we consider the change of sorption amount in elements $E_{1}$ and $E_{2}$ during the procedure stated above. As shown in Figure 4(a), upon adsorption, sorption amount in $E_{2}$ increases from the beginning as it is open. In contrast, no adsorption happens in $E_{1}$ as it is in a closed state. With increasing $\mathrm{RH}$, the swelling of element $\mathrm{E}_{2}$ stretches element $\mathrm{E}_{1}$ and its pore size becomes larger than the critical pore radius $\left(R>R_{\mathrm{C}}\right)$ so that $\mathrm{E}_{1}$ switches from closed to open state at $\mathrm{RH}_{1}$ and the sorption process starts. Note that a jump of sorption amount in $E_{1}$ occurs at this point from 0 to a finite value according to Eq. (1) as $\mathrm{RH}$ is not zero. Upon desorption, $\mathrm{E}_{1}$ remains open until $\mathrm{RH}_{2}$ and then switches from open back to closed. Note that $\mathrm{RH}_{2}<\mathrm{RH}_{1}$ as adsorbed molecules in $\mathrm{E}_{1}$ are difficult to remove. Here, for simplicity, we assume that $\mathrm{RH}_{2}$ equals 0 , meaning that elements $\mathrm{E}_{1}$ and $E_{2}$ desorb in a similar behavior following Eq. (1) (a critical discussion on this simplified closing mechanism will be presented in the discussion section). This qualitative image resembles the mechanism found in molecular simulations showing that water molecules tend to remain in newly generated adsorption sites during desorption until very low RH due to the high binding energy of $\mathrm{HB}^{\mathrm{CW}}$. As a result, the sorption process of element $\mathrm{E}_{1}$ is hysteretic, which finally contributes to 
sorption hysteresis of the total pore system. In brief, there are some elements in the porous system initially non-sorptive (closed) thus reversible. However, the neighboring elements make them switch between sorptive (open) and non-sorptive (closed) by mechanical interaction (stretching and compressing). As a result, the system exhibits a pronounced hysteresis loop.
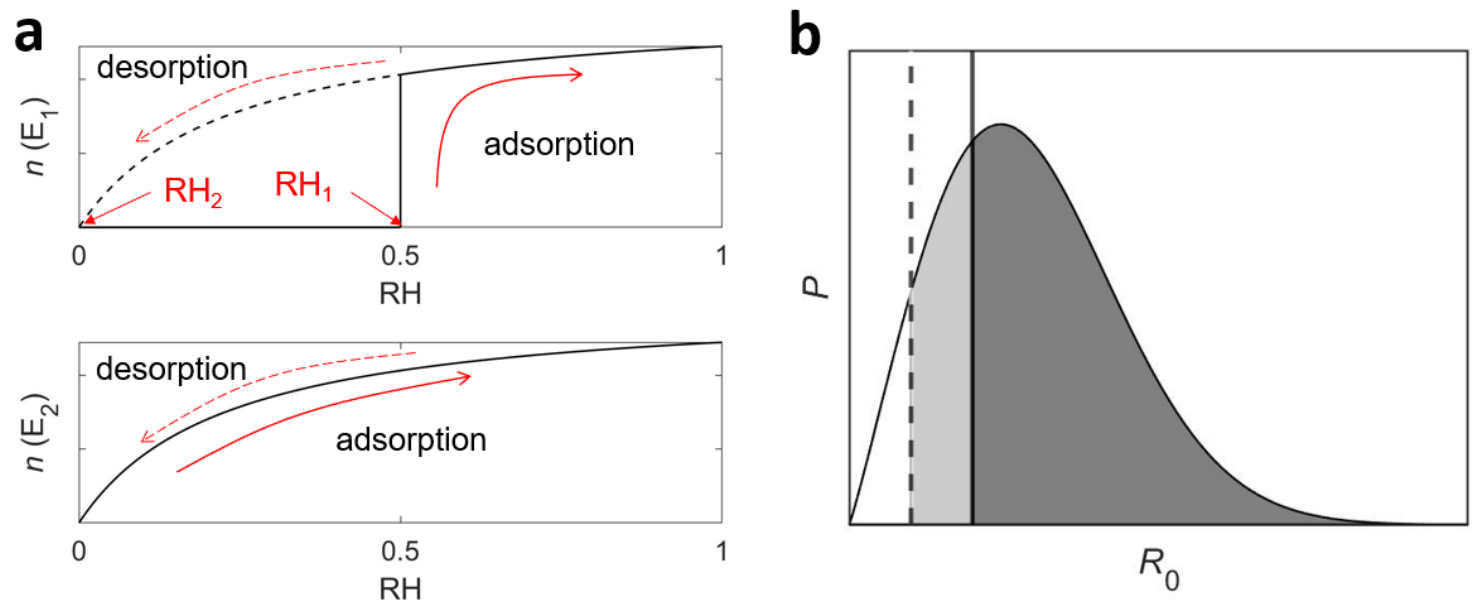

Figure 4 (a) Sorption isotherms of initially closed $\left(E_{1}\right)$ and open $\left(E_{2}\right)$ pores. The sorption isotherm of $\mathrm{E}_{2}$ is reversible (lower) as it remains open upon both adsorption and desorption. The sorption isotherm of $E_{1}$ is hysteretic (upper) as it is opened by neighboring pores during adsorption but remains open during desorption. (b) Population dependence of open and closed pores upon straining. The dark grey area represents the initial open pores, while the light grey area represents the additional pores opened during straining of the material.

Consider now the whole sets of elements in a pore system with a certain pore size distribution, PSD, as shown in Figure 4 (b). Initially, all the pores with a pore size above $R_{\mathrm{C}}$ are open, the population of which is the integration of PSD from $R_{\mathrm{C}}$ to infinity (dark grey area). After deformation, the swelling strain enlarges the pore size and shifts the pore size distribution rightward, bringing more pores initially below $R_{\mathrm{C}}$ to cross over the line and join the population of open pores. The effect of a right shift of pore size distribution is equivalent to a left shift of the critical value from $R_{\mathrm{C}}$ to $R_{\mathrm{C}} /(1+S \varepsilon)$ as shown in Figure 4(b).

\subsection{Poromechanics}

The mechanism given above involves sorption and deformation. Thus, the corresponding constitutive relationships must be specified and the coupling between sorption and deformation needs to be defined. In this work, we address these aspects within the framework of poromechanics. In conventional poromechanics, porosity is accounted for while pore size is not explicitly modeled. In our case, however, the pore size is crucial to properly model sorption hysteresis as it is the key 
parameter controlling the open/close status of the pores. Thus, the pore size must be included in the constitutive relationships. To do so, we employ a domain theory and divide the pore system into a collection of elements as shown in Figure 5(a). Each element is assigned with a pore size as shown in Figure 5(b). In other words, all the pores within the same element are assumed to have the same pore size. An advantage of this strategy for assigning each element one single pore size is that the element size can be chosen independently of the pore size, which simplifies the discretization process and allows embedding the DDM model at larger scales. In addition, all the pores in the same element are assumed to display the same behavior, i.e., they undergo the same strain and change their states under the same condition. For simplicity, we assume that all the elements have the same porosity so that they can be modeled with the same poromechanics equation. One should be aware that this means that the number of pores within each element is not necessarily the same. Then, as usually done in domain theory, the PSD of the system can be attained by adjusting the population of elements. As each element is assigned a specific pore size, it is easy to determine the open/closed state of the element. For elements with a current pore size larger than $R_{\mathrm{C}}$, sorption happens and coupling between sorption and mechanics is considered based on poromechanics as described below. Otherwise, no sorption occurs and only the mechanics is considered.

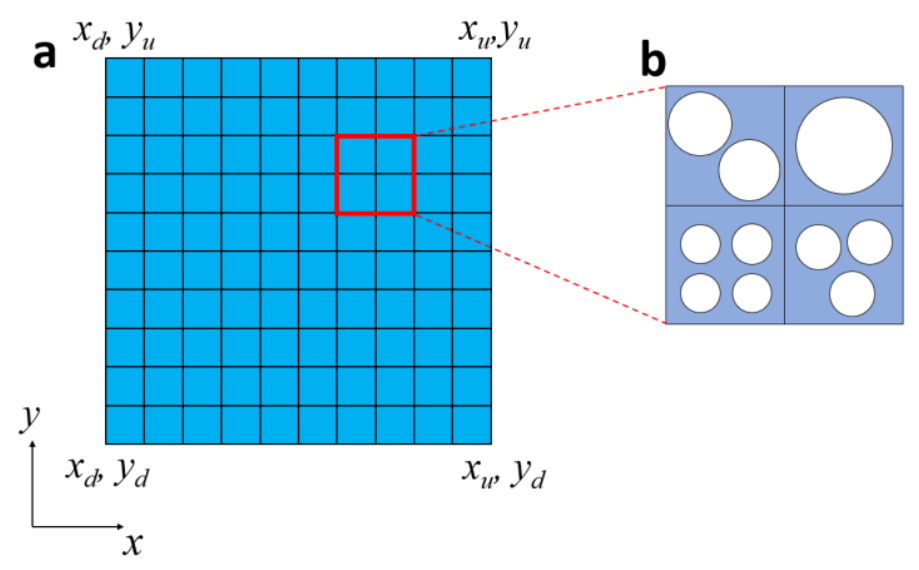

Figure 5 Schematic representation of (a) the porous material decomposed into a collection of spatial-distributed elements where (b) All elements have an identical porosity.

We model the coupling of sorption and deformation of the open element with the help of poromechanics. In agreement with molecular simulations on AC, we follow the assumption made by Kulasinski et al. ${ }^{39}$ and Zhang ${ }^{38}$ that the coupling between sorption and deformation is isotropic and the stress deviator is unaffected. With these assumptions, the grand potential density $\omega$ writes: 


$$
\mathrm{d} \omega=-s \mathrm{~d} T+\sigma \mathrm{d} \varepsilon+n \mathrm{~d} \mu
$$

where $s, \sigma$ and $n$ are the entropy density, volumetric stress and sorption amount respectively, while $T, \varepsilon$ and $\mu$ are temperature, strain and chemical potential. Note that in Eq. (3) we do not distinguish between the liquid and gas phase of adsorbed water molecules as other researchers ${ }^{54,55}$. This is mainly due to the small pore size of the polymer, which is on the order of the range of molecular interactions, and therefore all water molecules in a pore interact with the atoms of the polymer matrix. As one pore can only accommodate several water molecules, the basic concepts of specific surface of the pores as well as adsorbed liquid film become ambiguous. Thus, we treat the adsorbed water molecules as a whole and demonstrate the adsorbed amount by a single parameter $n$. This is consistent with the poromechanics method proposed by Brochard et $\mathrm{al}^{36}$ addressing the sorptioninduced deformation in microporous media. The Maxwell relation leads to

$$
\left.\frac{\partial \sigma}{\partial \mu}\right|_{\varepsilon, T}=\left.\frac{\partial n}{\partial \varepsilon}\right|_{\mu, T}
$$

Integration of the left and right hand sides of Eq. (4) with respect to the chemical potential $\mu$ gives

$$
\sigma=\sigma_{0}-\left.\frac{\partial}{\partial \varepsilon}\left[\int_{-\infty}^{\mu} n \mathrm{~d} \mu\right]\right|_{T}=\sigma_{0}-\sigma_{s}
$$

where $\sigma_{0}$ is the volumetric stress that prevails when there is no fluid in the pores, $\sigma_{\mathrm{s}}$ is the sorptionrelated stress, which represents the impact of sorption on the stress state of the system and is coined 'sorption stress' in this work. In the following, stress and strain deviators are not considered, since it is assumed that sorption induced deformation is only volumetric in homogeneous isotropic materials. Unless notified, the terms stress and strain refer to volumetric stress and strain respectively. To apply Eq. (5), the sorption isotherm and mechanical constitutive relationship of the dry material, i.e. $n \sim n(\mu, \varepsilon)$ and $\sigma_{0} \sim \sigma_{0}(\varepsilon)$, have to be specified.

In this work we assume an ideal condition that the polymers are completely dry (without any residue water) at the dry state, and we take the strain of the dry state as zero. For cases with pre-strains or residue water molecules, one can simply add the pre-strain / residue sorption amount to the total strain / sorption amount without changing the following derivation. For the sorption isotherm, we first consider the sorption isotherm at zero strain, i.e. $n \sim n(\mu, \varepsilon=0)$. It has been shown by molecular simulations that the nanoporous polymer at zero strain (no deformation during sorption) yields a Type-I isotherm ${ }^{14}$, which is consistent with the experiments carried out on other stiff materials 
with very limited sorption-induced deformation. We adopt the Langmuir isotherm, which is suitable to describe the Type-I isotherm, i.e.

$$
\left.n\right|_{\varepsilon=0}=\frac{n_{0} B p}{1+B p}
$$

where $n_{0}$ and $B$ are sorption parameters. $n_{0}$ defines the sorption capacity while $B$ describes the rate of sorption amount increase upon increasing vapor pressure $p$.

The strain may affect significantly the sorption process in many aspects. For instance, strain can change the distance between the solid and fluid atoms, thus both the rate and capacity of sorption can be affected. For simplicity, only the effect of strain on sorption capacity is considered here. The sorption amount is assumed to be proportional to the current porosity, as follows

$$
n=\frac{\phi}{\phi_{0}} \frac{n_{0} B p}{1+B p} .
$$

Following ${ }^{36}$, the porosity $\phi$ is related to the strain by $\phi=\phi_{0}(1+C \varepsilon)$, where $C$ is a coupling constant and the sorption isotherm dependence on strain can be written as

$$
n=(1+C \varepsilon) \frac{n_{0} B p}{1+B p}
$$

Note that the vapor pressure $p$ is related to the chemical potential $\mu$ by

$$
\mathrm{d} \mu=\frac{R_{g} T}{p} \mathrm{~d} p
$$

where $R_{g}$ and $T$ are the ideal gas constant and temperature respectively. Substituting Eqs.(8),(9) into Eq.(5), the sorption stress $\sigma_{\mathrm{s}}$ can be determined as

$$
\sigma_{s}=C R_{g} T n_{0} \ln (1+B p)
$$

According to Eq. (10), the sorption stress depends monotonically on the vapor pressure. However, the sorption stress is derived based on the homogenization of pores with different sizes. One should note that, at single micropore level, sorption stress generally exhibits an oscillatory behavior ${ }^{56-58}$. We now consider the mechanical constitutive relationship of the dry porous material. Since Straining of the material increases the inter-chain distance, increases the porosity and also weakens the polymer, the tangential modulus $K$ is assumed to decay with the porosity which can be expressed as: 


$$
\mathrm{d} \sigma_{0}=K \mathrm{~d} \varepsilon=K_{0} \exp \left[-b\left(\phi-\phi_{0}\right)\right] \mathrm{d} \varepsilon
$$

where $K_{0}$ is the bulk modulus at dry state with zero strain, while $b$ is a parameter controlling the mechanical weakening of the material. Note that $\phi$ in Eq. (11) depends on strain $\varepsilon$ as $\phi=\phi_{0}(1+C \varepsilon)$ so that the bulk modulus $K$ is a function of strain $\varepsilon$. Combining Eqs. (5),(8) and (11) allows predicting and modeling the coupling between sorption and deformation in the open elements. For the closed element, only mechanics is involved as no sorption occurs. As a result, the behavior of the closed element can be fully characterized by Eq. (11). With these relationships, all constitutive equations required for the DDM are available.

\subsection{Parameters Determination}

There are four groups of parameters to be determined in the proposed DDM: parameters describing the pore size distribution (PSD), structure-related parameters $\left(\phi_{0}, C\right.$ and $\left.S\right)$, the mechanics-related parameters $\left(K_{0}\right.$ and $\left.b\right)$ and the sorption-related parameters $\left(B\right.$ and $\left.n_{0}\right)$. The accuracy of DDM relies on careful determination of these parameters from the molecular simulation results. First, we look at the description of the PSD. As the switch of the elements from closed to open (or reverse) relies on pore size, an accurately determination of the initial PSD is needed. The initial PSD is extracted from a molecular model of amorphous cellulose previously published in Ref. ${ }^{14}$. Here we adopt the method suggested by Ref. ${ }^{15}$ to determine the PSD. The probability $f\left(R_{0}\right)$ used as PSD is defined as $f=\mathrm{d} F / \mathrm{d} R_{0}$ where $F$ is the fraction of pore volume with a pore radius below $R_{0}$. In practice, $f$ is described with a three-parameter Weibull distribution, i.e.

$$
f\left(R_{0}\right)=\frac{\beta}{\eta}\left(\frac{R_{0}-R_{\text {shift }}}{\eta}\right)^{\beta-1} e^{-\left(\frac{R_{0}-R_{\text {shift }}}{\eta}\right)^{\beta}}
$$

where $\beta>0$ is the shape parameter, $\eta>0$ is the scale parameter and $R_{\text {shift }}$ is the shifting parameter of the distribution. The obtained PSD is plotted in Figure 6 in comparison with the molecular simulation results. The solutions of DDM in the next sections are based on this pore size distribution function. 


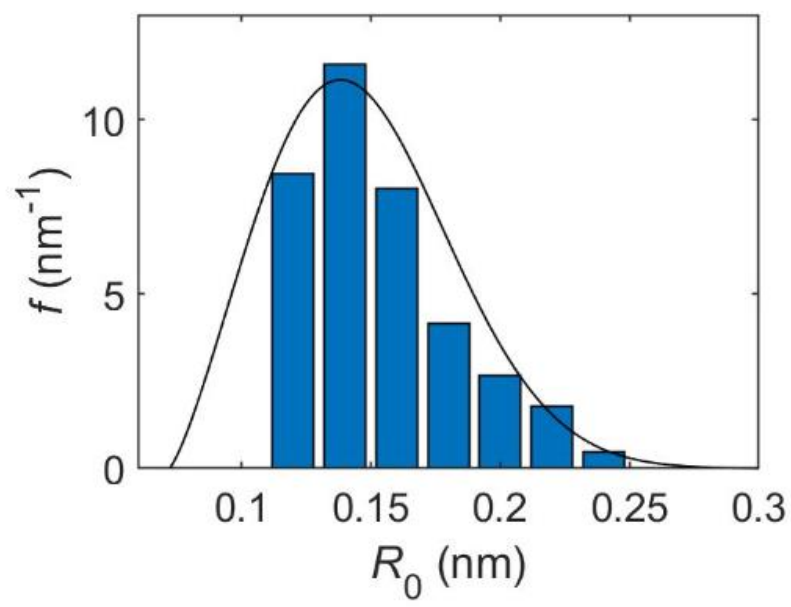

Figure 6 PSD determined based on molecular simulation results (blue histogram). The black line is the PSD based on a three-parameter Weibull distribution fitted to the molecular simulation data.

There are three structure-related parameters in the DDM: $\phi_{0}, C$ and $S$. The initial porosity at dry state $\phi_{0}$ is determined in the molecular model with a probe with a radius of $0.08 \mathrm{~nm}$. For $C$, we follow the assumption in Ref. ${ }^{36}$ that the solid part is rigid and the strain of the system comes from the change in porosity, which gives $C=1 / \phi_{0}$. For $S$, we consider its relationship with $C$ based on a spherical pore assumption. Note that $C \varepsilon$ and $S \varepsilon$ are the volumetric and one-dimensional change of the pore respectively. It is straightforward to write

$$
\frac{\phi-\phi_{0}}{\phi_{0}}=C \varepsilon=(1+S \varepsilon)^{3}-1
$$

Neglecting the contribution of the high order term in $\varepsilon$, we get $S \approx C / 3$. Thus, $S$ is taken as one third of $C$ in the model.

Table 1 Parameters used for the poromechanics model

\begin{tabular}{|c|c|c|c|c|c|c|c|}
\hline parameter & $n_{0}$ & $B$ & $K_{0}$ & $b$ & $\phi_{0}$ & $C$ & $S$ \\
\hline units & $\left(\mathrm{nm}^{-3}\right)$ & $\left(\mathrm{Pa}^{-1}\right)$ & (GPa) & - & - & - & - \\
\hline value & 3.58 & 0.043 & 4.01 & 11.21 & 0.16 & 6.25 & 2.06 \\
\hline
\end{tabular}

For the mechanics-related parameters, $K_{0}$ and $b$, that control the stress-strain relationship of the solid, we first extract the nonlinear stress-strain curve for the dry material from molecular simulations and then fit it with Eq. (11) allowing to determine $K_{0}$ and $b$. For the sorption-related 
parameters $n_{0}$ and $B$, we determine the sorption isotherm at zero strain (no deformation allowed) by molecular simulations and fit it with Eq. (6) to estimate $n_{0}$ and $B$. The determined parameters are listed in Table 1.

\subsection{Finite Element Solution}

According to Ref. ${ }^{32}$, the DDM can be solved using a Finite Element (FE) solver. Practically, one can assign an initial pore size to each element of the computation domain of the FE method. Depending on the pore size, the element states are classified as open or closed and the corresponding constitutive relationships are applied. The state of closed elements changes into open during adsorption based on the current pore size and switches back to closed upon full drying in desorption. Following this approach, elements with different pore sizes undergo different strains. As these elements are assembled together mechanically, they will certainly impact each other mutually via mechanical interactions. In this way, the local deformation effect is included in our model.

A 2-D FE model is built with the help of ANSYS 19.0 under the assumption of plane stress. A square geometry with unit length is built to represent the solution domain of the nanoporous polymers discretized in $20 \times 20$ elements. A mesh dependent study on different degrees of discretization is given in Appendix. For each element, the 2-D 4-node plane stress element (PLANE182) is adopted. As shown in Figure 5, the boundaries of the FE model include 4 lateral sides. The boundary conditions for displacement include two parts. A first part is the removal of degree of freedom at the left side $x=x_{\mathrm{d}}$ in the $x$-direction and at the bottom side $y=y_{\mathrm{d}}$ in the $y$ direction by assigning zero displacement values in the respective directions:

$$
\begin{aligned}
& \left.u_{x}\right|_{x=x_{\mathrm{d}}}=0 \\
& \left.u_{y}\right|_{y=y_{\mathrm{d}}}=0
\end{aligned}
$$

The second part consists of imposing the same displacement in the $y$-direction at the top side $x=x_{\mathrm{u}}$ and the same displacement in the $x$-direction at the left side $y=y_{\mathrm{u}}$ using coupled degrees of freedom:

$$
\begin{aligned}
& \left.u_{x}\right|_{x=x_{u}, y \neq y_{u}}=\left.u_{x}\right|_{x=x_{u}, y=y_{u}} \\
& \left.u_{y}\right|_{x \neq x_{u}, y=y_{u}}=\left.u_{x}\right|_{x=x_{u}, y=y_{u}}
\end{aligned}
$$


With the boundary conditions defined above, all four lateral sides of the FE model remain straight upon deformation. The coupled behavior implemented in the FE model is nonlinear. The nonlinearity comes from two aspects: (1) the material nonlinearity coming from the mechanical weakening behavior of single element (bulk stiffness dependent on strain); (2) the system nonlinearity stemming from the change of population of open/closed elements. This nonlinear problem in FE is solved iteratively using the Newton-Raphson method.

\subsection{Analytical Solution}

The advantage of a DDM model implemented in FE is that it solves numerically the strain field of the domain and, from this, the change of pore size of each element is determined. The solution of the strain field can be further simplified by introducing the equal-strain condition assuming that the strain field is uniform over the whole domain. This allows deriving an analytical solution for the coupled behavior of deformation and sorption. For a pore system with a distribution function of the initial pore size $f\left(R_{0}\right)$ at dry state, the pore size distribution changes into $g(R)$ due to deformation after subjecting the sample to a vapor pressure $p$ and an external mechanical loading $\sigma_{\text {ext }}\left(R_{0}\right.$ and $R$ are the pore sizes before and after deformation). Elements in this system can be either open or closed, which can be represented by a state variable $i=\mathrm{o}$ and $i=\mathrm{c}$ respectively. Force equilibrium at the scale of the pore system requires:

$$
\frac{1}{V_{\mathrm{t}}}\left\{\int_{i=\mathrm{o}}\left[\sigma_{0}(\varepsilon)-\sigma_{\mathrm{s}}\right] \mathrm{d} V+\int_{i=\mathrm{c}} \sigma_{0}(\varepsilon) \mathrm{d} V\right\}=\sigma_{\text {ext }}
$$

where $V_{\mathrm{t}}$ is the total volume of the system and $\sigma_{\mathrm{ext}}$ is the external stress loading, which is zero for

the free swelling case. $\int_{i=0} \square \mathrm{d} V$ and $\int_{i=c} \square \mathrm{d} V$ represent integration over the volume of open and closed elements, respectively. Upon adsorption, the number of open elements changes because of the change in pore size. By introducing $\int \square \mathrm{d} V / V_{t}=\int \square g(R) \mathrm{d} R$ into Eq. (16), the latter equation becomes

$$
\int_{R \geq R_{\mathrm{c}}}\left[\sigma_{0}(\varepsilon)-\sigma_{\mathrm{s}}\right] g(R) \mathrm{d} R+\int_{R<R_{\mathrm{c}}} \sigma_{0}(\varepsilon) g(R) \mathrm{d} R=\sigma_{\mathrm{ext}}
$$

Note that $R$ is a function of $\varepsilon$ as indicated in Eq. (2). Using Eq. (2) to transform the integration limits in Eq. (17), we get 


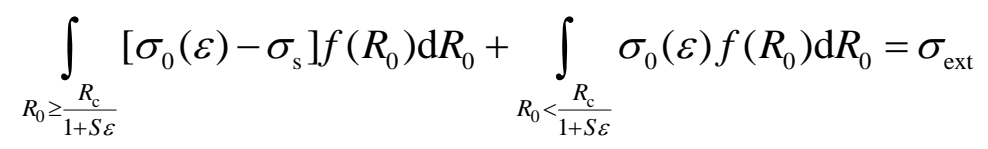

Note that the lower limit of integration on the initial pore size distribution $f\left(R_{0}\right)$ in Eq. (18) is transformed from the critical pore size $R_{\mathrm{C}}$ to the nominal critical pore size $R_{\mathrm{CC}}(\varepsilon)=R_{\mathrm{C}} /(1+S \varepsilon)$, which means all the pores with initial pore sizes larger than $R_{\mathrm{CC}}$ are sorptive. For the swelling case, $\varepsilon$ is positive which leads to $R_{\mathrm{CC}}<R_{\mathrm{C}}$. This means that some pores with initial pore sizes lower than $R_{\mathrm{C}}$ are opened and become sorptive upon swelling. As the swelling strain increases, $R_{\mathrm{CC}}$ becomes smaller and more pores switch their state. Given the initial pore size distribution of the system $f\left(R_{0}\right)$, the strain of the system along the adsorption branch can be analytically determined

$$
\begin{aligned}
& \varepsilon=-\frac{1}{b \phi_{0} C} \ln \left\{1-\left[\alpha(\varepsilon) C \ln (1+B p) n_{0}^{0} \phi_{0} R_{g} T+\sigma_{e x t}\right] b \phi_{0} C \exp \left(b \phi_{0}\right) / K_{0}\right\} \\
& \alpha(\varepsilon)=\int_{\frac{R_{c}}{1+S \varepsilon}}^{\infty} f\left(R_{0}\right) \mathrm{d} R_{0}
\end{aligned}
$$

For the desorption branch, as all the opened elements switch back to closed upon full drying at RH $=0$, the population of opened elements does not change until full drying. Thus, $\alpha$ does not dependent on strain and remains at the maximum value reached upon adsorption, i.e.,

$$
\alpha(\varepsilon)=\int_{\frac{R_{\mathrm{c}}}{1+S \varepsilon_{\max }}}^{\infty} f\left(R_{0}\right) \mathrm{d} R_{0}
$$

where $\varepsilon_{\max }$ is the maximum strain reached along the adsorption branch.

\section{Results}

The results of the DDM implemented in FE (DDM-FE) model are plotted as triangles in Figure 7. The system undergoes swelling during adsorption which leads to a shift of the nominal critical pore size value $R_{\mathrm{CC}}$ from $0.28 \mathrm{~nm}$ down to $0.0824 \mathrm{~nm}$ as shown in Figure 7(a). The sorption isotherms show good agreement with molecular simulation results at free swelling conditions (Figure 7(b)): type II sorption isotherm and hysteresis persisting over the full range of RH. Figure 7(c) compares the strain isotherms obtained by DDM-FE and MD simulations, showing that they are in good agreement. Figure 7(d) compares the relationship between strain and sorption amount. The results of DDM-FE show a linear function from low to high sorption amount region, but with an initial convex part in the very low sorption amount region. As shown by molecular simulations, this 
behavior is due to the presence of free pore space being initially filled at low $\mathrm{RH}$ - thus an increase in sorption amount does not cause much swelling. Once the initial porosity is filled, the increase in sorption amount is linked to the increase in pore space caused by swelling. In this regime, the relationship between strain and sorption amount becomes linear. The agreement between DDM-FE and molecular simulations shows that this microscopic mechanism is successfully captured in DDM-FE. However, it is worth noting that there exists a small hysteresis between the swelling strain plotted versus sorption amount, which has not been found in molecular simulations previously. Specifically, at the same strain, the system shows a slightly higher sorption amount upon desorption. This can be qualitatively explained by more pores being in the open state and staying filled during desorption. Thus, sorption happens in more elements at the same strain in desorption compared to adsorption, which finally gives a slightly higher sorption amount. However, the hysteresis between strain and sorption amount shown by DDM-FE is very limited compared to that in sorption and strain isotherms. 

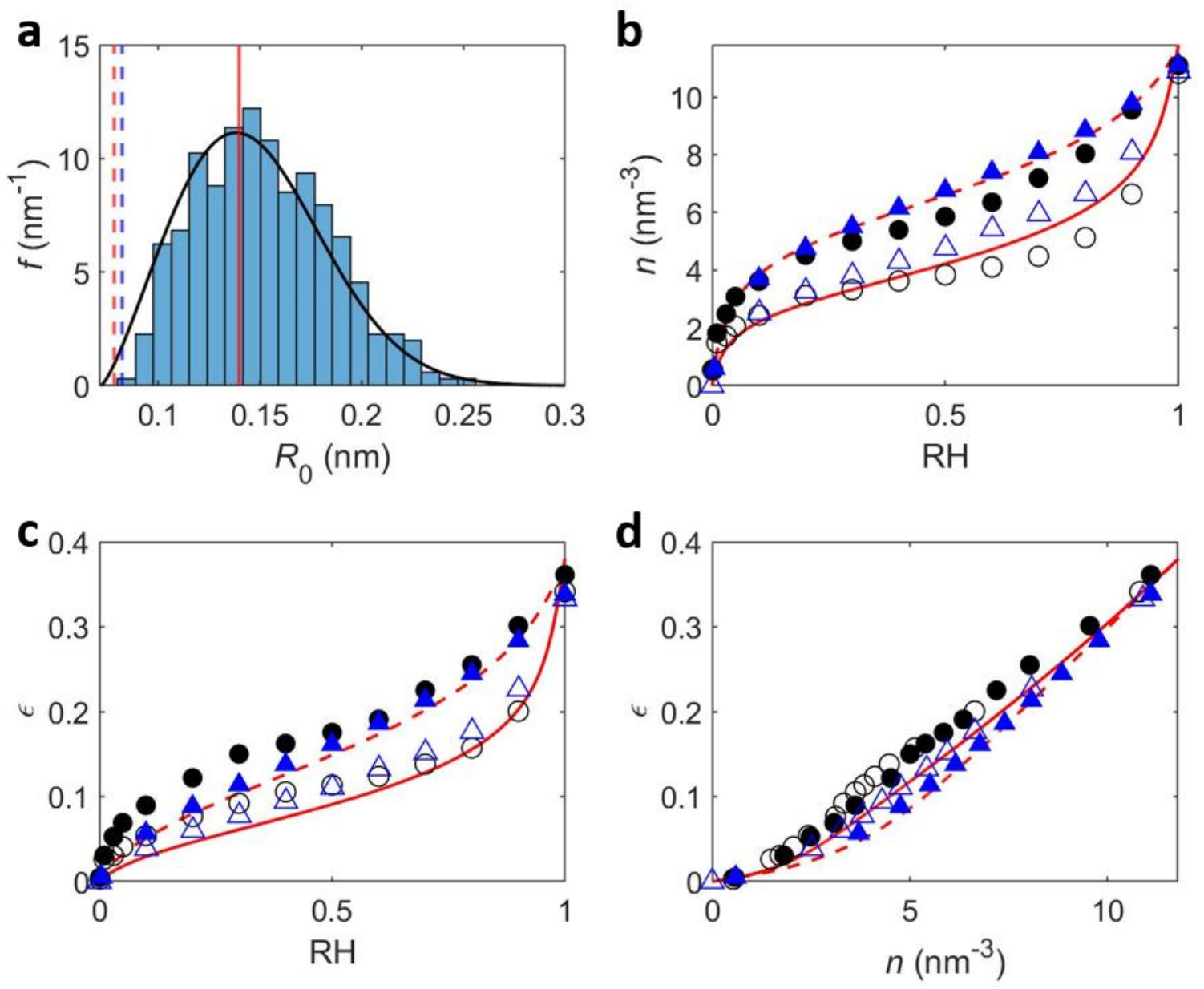

Figure 7 (a) Change of nominal critical pore size $R_{\mathrm{CC}}$ in DDM. PSD used in DDM is depicted by the black solid line. Pore sizes generated by the PSD are categorized by the blue histogram. The red solid vertical line represents the critical pore size at the initial (dry) state. The blue and red dashed vertical lines represent the critical pore size at the saturated state of the DDM-FE model and analytical model respectively. (b) Sorption isotherms determined by DDM in comparison with molecular simulations. (c) Strain isotherms determined by DDM in comparison with molecular simulations. (d) Strain-sorption amount relationships determined by DDM in comparison with molecular simulations. For (b-d), the blue triangles represent the DDM-FE solution, with open and closed dots representing the adsorption and desorption respectively. The red line represents the analytical solution, with solid and dashed lines representing the adsorption and desorption respectively. The adsorption and desorption data of molecular simulations are plotted as open and closed black dots.

Figure 8 shows the evolution of the open/closed state of elements upon adsorption process. As RH increases, the swelling strain increases which leads to more elements in the open state as shown in Figure 8. Moreover, it also shows that the switch of state of an element follows a pattern that is not totally random. The grey 'islands' lose their territory from the borders inwards, which indicates that the switch of the elements from closed to open state first happens at the border. Indeed, as the 
switch can only happen with the help of the stretching effect imposed by neighboring open elements, it is easy to understand that the closed elements at the boundary are subjected to a higher mechanical stretching effect compared to those embedded within the regions of closed elements.
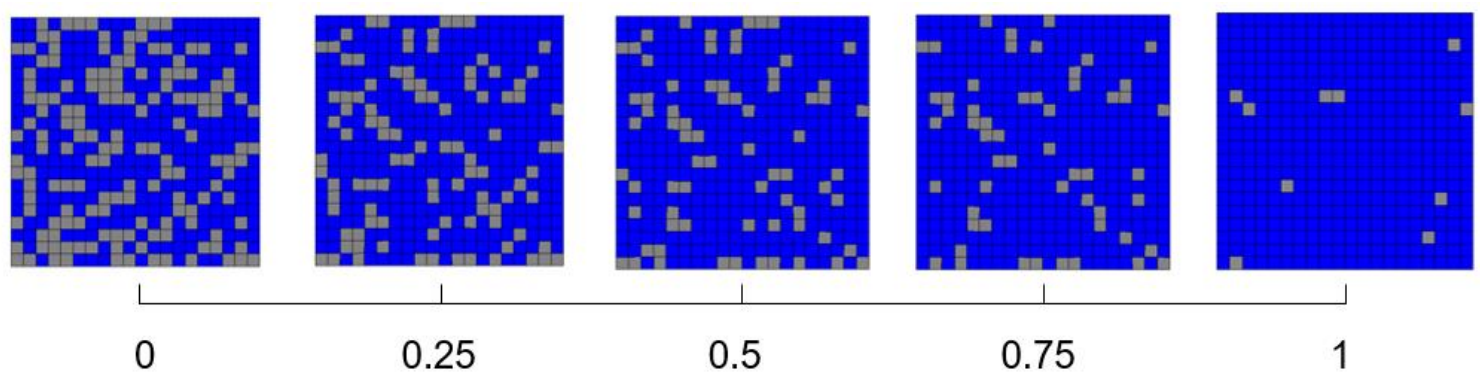

0.25

\section{5}

0.75

$\mathrm{RH}$

Figure 8 Distribution of closed (grey) and open (blue) elements at different RH obtained by DDMFE.

Figure 9 shows the contour plots of the distribution of sorption amount at three occurrences in both adsorption and desorption branches. The distribution is more heterogeneous upon adsorption than desorption at the same RH as shown in Figure 9. This can be attributed to the elements opened during adsorption remaining open during desorption, which corresponds to the mechanism found in molecular simulations (i.e. water tends to remain in between the cellulose chains after breaking the $\mathrm{HB}^{\mathrm{CC}}$ ). This microscopic picture is consistent with the density map of hydrogen bonds reported in Ref. ${ }^{14}$, which shows that there are less clustered water molecules, i.e. more evenly distributed, attached to cellulose chains during desorption. 


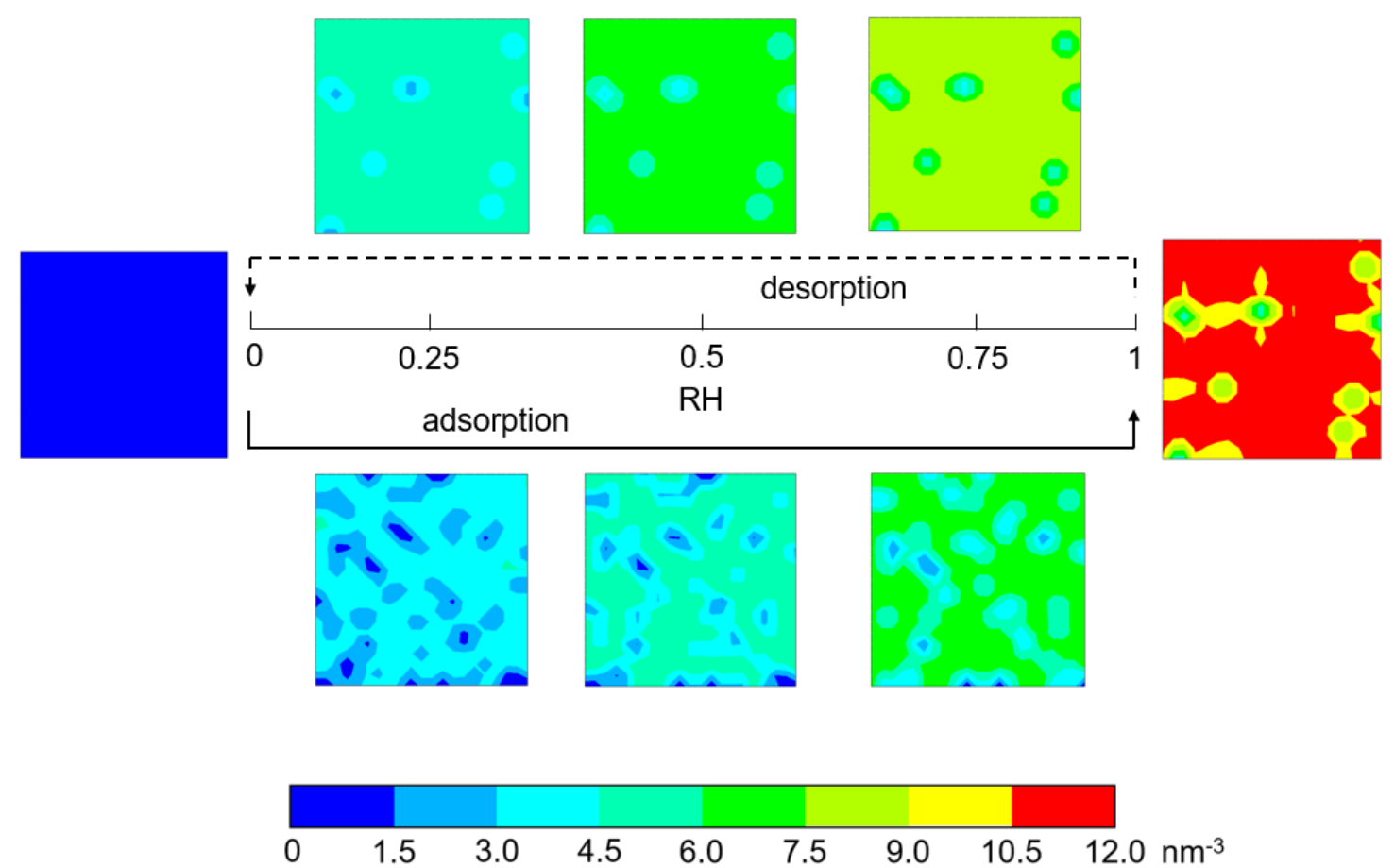

Figure 9 Comparison of DDM-FE sorption amount distributions between adsorption and desorption at $\mathrm{RH}=0.25,0.5$ and 0.75 . Desorption branch shows higher sorption amount at the same $\mathrm{RH}$, with a more heterogeneous water distribution.

The results based on the analytical model (equal-strain assumption) are plotted in Figure 7 in comparison with the molecular simulations and FE solutions. Figure 7(a) shows that the shift of the nominal critical pore size value $R_{\mathrm{CC}}$ for the analytical model is in close agreement to that of the DDM-FE model, changing from $0.28 \mathrm{~nm}$ down to $0.0785 \mathrm{~nm}$ versus $0.0824 \mathrm{~nm}$ As shown in Figure 7(b) and (c), the sorption and strain isotherms acquired from the analytical model agree well with molecular simulations - both for the adsorption and desorption branches. Finally, Figure 7(d) shows a small hysteresis between adsorption and desorption as already noted in the DDM-FE results. Comparison between the analytical solutions and DDM-FE results in sorption and strain isotherms (Figure $7(\mathrm{c}, \mathrm{d})$ ) shows that the analytical solutions tend to underestimate the sorption amount and strain along the adsorption branch, while the agreement is better along the desorption branch. Indeed, the system can be viewed as a composite system composed of two different elements: the open and closed elements, though heterogeneity originates from the internal load heterogeneity (as sorption stress acts only on the open element) instead of the material heterogeneity. The equal strain assumption employed in this work basically provides the Voigt average, ${ }^{59}$ which corresponds to 
the upper bound of the stiffness. In other words, the equal strain assumption tends to slightly overestimate the stiffness compared to the DDM-FE solution. As a result, the analytical solutions yield slightly lower strain, leading to lower sorption amount. Along the desorption branch, as most of the elements are opened, the system becomes less heterogeneous. Thus, the DDM-FE model for the desorption is much closer to the uniform assumption made in the analytical model compared to that for the adsorption branch (Figure 9).

\section{Discussion}

\subsection{Closing Mechanism during desorption}

As mentioned before, the open/closed state of the elements in DDM has been conceived as corresponding to the breakage/reformation of $\mathrm{HB}^{\mathrm{CC}}$ in molecular simulations. The opening process is reflected by the accessibility of pores as a function of strain in the adsorption branch, which corresponds to breakages of $\mathrm{HB}^{\mathrm{CC}}$. In desorption, it is assumed that all elements only close at zero RH. One way of validating this mechanism in DDM is to compare the opening/closing process to the change of $\mathrm{HB}^{\mathrm{CC}}$ in molecular simulations. Or otherwise stated the number of elements remaining closed should be consistent with the number of $\mathrm{HB}^{\mathrm{CC}}$. Here we define the relative ratio of closed elements and $\mathrm{HB}^{\mathrm{CC}}$ as

$$
X=\frac{Y-Y_{\min }}{Y_{\max }-Y_{\min }}
$$

where $X$ is the relative ratio, $Y$ is the parameter of interest, which is the number of closed elements in DDM or the number of $\mathrm{HB}^{\mathrm{CC}}$ in molecular simulations. $Y_{\min }$ and $Y_{\max }$ correspond to the minimum and maximum value taken by $Y$. The dependence of the closed elements on sorption amount obtained from analytical solution in adsorption branch is compared with $\mathrm{HB}^{\mathrm{CC}}$ from molecular simulations in Figure 10(a). A good agreement is found. This indicates that the concept of open pores in DDM captures well the breakage of $\mathrm{HB}^{\mathrm{CC}}$ in molecular simulations.

In this work, the opened elements are assumed to remain open along the desorption branch until full drying. In other words, all the opened elements close together at $\mathrm{RH}=0$, which corresponds to the red dashed line in Figure 10(a). This assumption maximizes the hysteresis effect and can be deemed as a theoretical bound addressing hysteresis. However, the closing mechanism of the opened pores is probably much more complex, and one may assume a more gradual closing process of pores in desorption as indicated by the $\mathrm{HB}^{\mathrm{CC}}$ reforming gradually according to molecular simulations. 

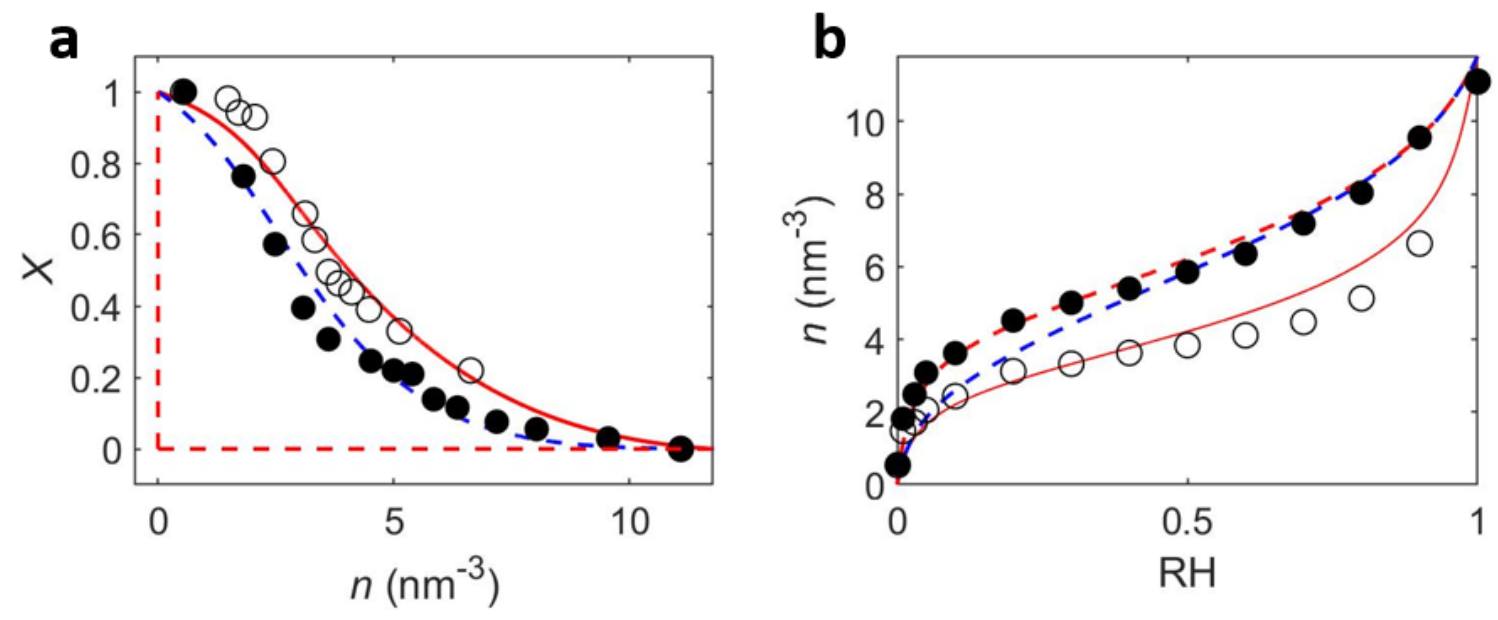

Figure 10 (a) Relative ratio of closed pores under different closing critiria in comparison with relative ratio of $\mathrm{HB}^{\mathrm{CC}}$ in molecular simulations. (b) Sorption isotherms under different closing critieria in comparison with moelcular simulations. The open and closed dots represent results of adsorption and desorption from molecular simulations. The red solid line represents the adsorption results from analytical solution. The red dashed line represents the desorption results from analytical solution assuming pores are closed upon full drying. The blue dashed line represents the desorption results from analytical solution assuming pores are closed following the closing criteria set by Eq.(22).

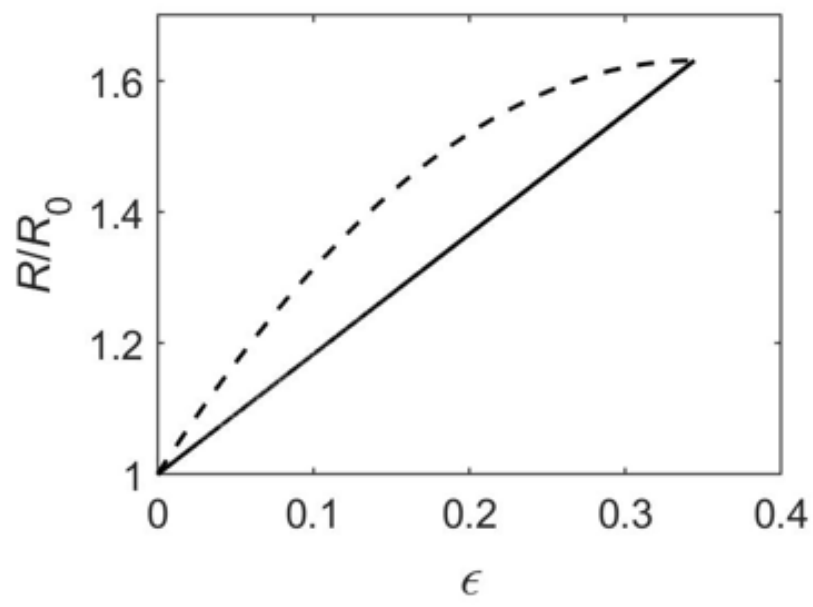

Figure 11 Dependence of the pore size $R$ on strain $\varepsilon$. The solid and dashed lines represent the adsorption and desorption respectively. $R_{0}$ is the unstrained pore size at dry state.

Upon adsorption we assumed a linear dependence of pore size $R$ on strain as indicated in Eq. (2) (solid line in Figure 11). Based on the molecular simulation results, we propose for desorption another relation describing the effect of the presence of water molecules on the delay in pore closing. 
Assuming that fewer pores close at high sorption amount while more pores close at low sorption amount, a nonlinear dependence of pore size $R$ on strain is proposed (dashed line in Figure 11). Here, we assume the pore size upon desorption to be a second-order function of strain, i.e.,

$$
\left.R(\varepsilon)\right|_{\text {desorption }}=A_{1} \varepsilon^{2}+A_{2} \varepsilon+A_{3}
$$

The coefficients $A_{1}, A_{2}$ and $A_{3}$ cannot be determined arbitrarily as Eq. (22) has to remain consistent with Eq. (2) in the dry and saturated states, i.e.,

$$
\left\{\begin{array}{c}
\left.R(0)\right|_{\text {desorption }}=R_{0} \\
\left.R\left(\varepsilon_{\text {max }}\right)\right|_{\text {desorption }}=R_{0}\left(1+S \varepsilon_{\text {max }}\right)
\end{array}\right.
$$

where $\varepsilon_{\max }$ is the strain at $\mathrm{RH}=1$ which is the maximum strain reached in the free swelling condition. Moreover, we assume that $\left.R(\varepsilon)\right|_{\text {desorption }}$ is a monotonically increasing function over the range [0, $\left.\varepsilon_{\max }\right]$, i.e.,

$$
\left.\frac{\mathrm{d} R}{\mathrm{~d} \varepsilon}\right|_{\text {desorption }} \geq 0 \text { for } \varepsilon \in\left[0, \varepsilon_{\max }\right]
$$

Considering all the constraints set in Eqs. (23) and (24), $A_{1}, A_{2}$ and $A_{3}$ can be determined by fitting the obtained relative ratio of closed elements in DDM to $\mathrm{HB}^{\mathrm{CC}}$ data in desorption with the help of least square algorithm.

The relative ratio of closed pores in desorption is plotted in Figure 10(a) as the blue dashed line. It shows that the population of closed pores given by this phenomenological closing model is consistent with that of $\mathrm{HB}^{\mathrm{CC}}$ in molecular simulations. However, Figure 10(b) shows that the agreement between sorption isotherms of the analytical model and molecular simulations is not improved by this enriched closing model compared to our primary assumption that all opened pores get closed at full drying. This indicates that the proper formulation of a physically-based closing mechanism remains an open question and calls for further research.

\subsection{Dependence of Hysteresis on Material Properties}

Hysteresis originates from the coupling between sorption and deformation. Thus, the sorption characteristics and mechanical properties will have significant impact on the hysteresis behavior. The DDM developed in this work is physically grounded, with mechanical and sorption properties explicitly included to allow studying the influence of different material properties on sorption hysteresis. For the study of these effects, a quantitative description of hysteresis is required. As shown in Figure 12, the 'backbone isotherm' is defined as the average curve of the adsorption and 
desorption isotherms. The area under the backbone is defined as $\mathrm{S}_{\mathrm{b}}$. As a measure for the degree of hysteresis, we use the area between adsorption and desorption curves $S_{h}$. The relative magnitude of hysteresis is expressed by the ratio of $\mathrm{H}=\mathrm{S}_{\mathrm{h}} / \mathrm{S}_{\mathrm{b}}$.

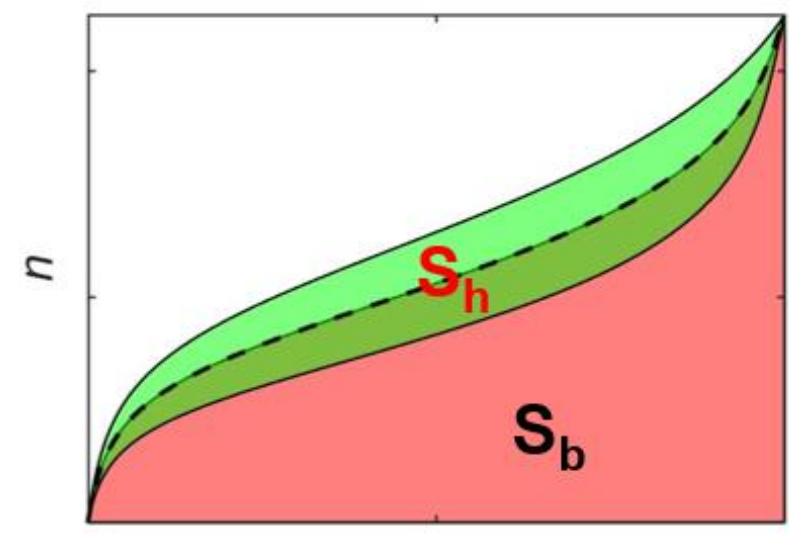

\section{$\mathrm{RH}$}

Figure 12 Characterization of sorption hysteresis in the adsorption isotherm $n(\mathrm{RH}) . \mathrm{S}_{\mathrm{h}}$ is defined as the area (green) between adsorption and desorption curves. The black dashed line is the backbone curve defined as the average of adsorption and desorption curves. $S_{\mathrm{b}}$ is defined as the area (red) under the backbone. Hysteresis is characterized by $\mathrm{H}=\mathrm{S}_{\mathrm{h}} / \mathrm{S}_{\mathrm{b}}$.
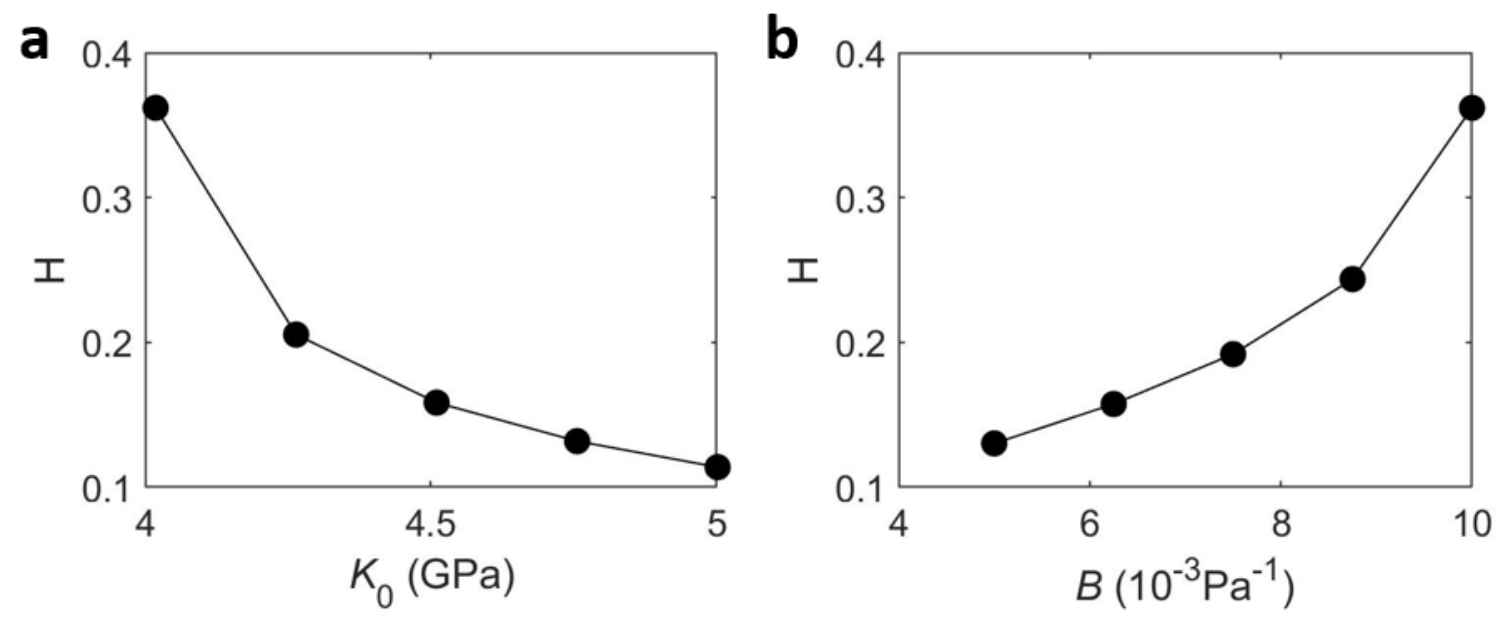

Figure 13 Dependence of sorption hysteresis $\mathrm{H}$ on initial modulus $K_{0}$ and sorption parameter $B$.

We study the influence of the bulk modulus $K_{0}$ from the mechanical side and sorption parameter $B$ from the sorption side on the hysteresis. A material with higher $K_{0}$ corresponds to a stiffer material, while a system with larger $B$ corresponds to stronger adsorbent-adsorbate interactions. The dependence of hysteresis characterized by $\mathrm{H}$ on $K_{0}$ and $B$ is plotted in Figure 13. It shows that $\mathrm{H}$ 
decreases with increasing $K_{0}$ and with decreasing $B$. In other words, a system with larger stiffness and lower adsorbent-adsorbate interaction strength tends to exhibit less sorption hysteresis.

The detailed sorption isotherms at different $K_{0}$ and $n_{0}$ are plotted in Figure 14. It shows that the sorption isotherm changes from type II to type I upon increasing $K_{0}$ and the hysteresis loop shrinks significantly. For a stiffer material, the magnitude of the swelling strain is smaller. As a result, there are fewer pores switching from closed to open state by the stretching effect of neighboring pores. Considering the fact that only the pores experiencing a switch between closed and open states contribute to the sorption hysteresis, the lower amount of newly opened pores for a stiffer material in adsorption branch finally leads to a smaller sorption hysteresis.
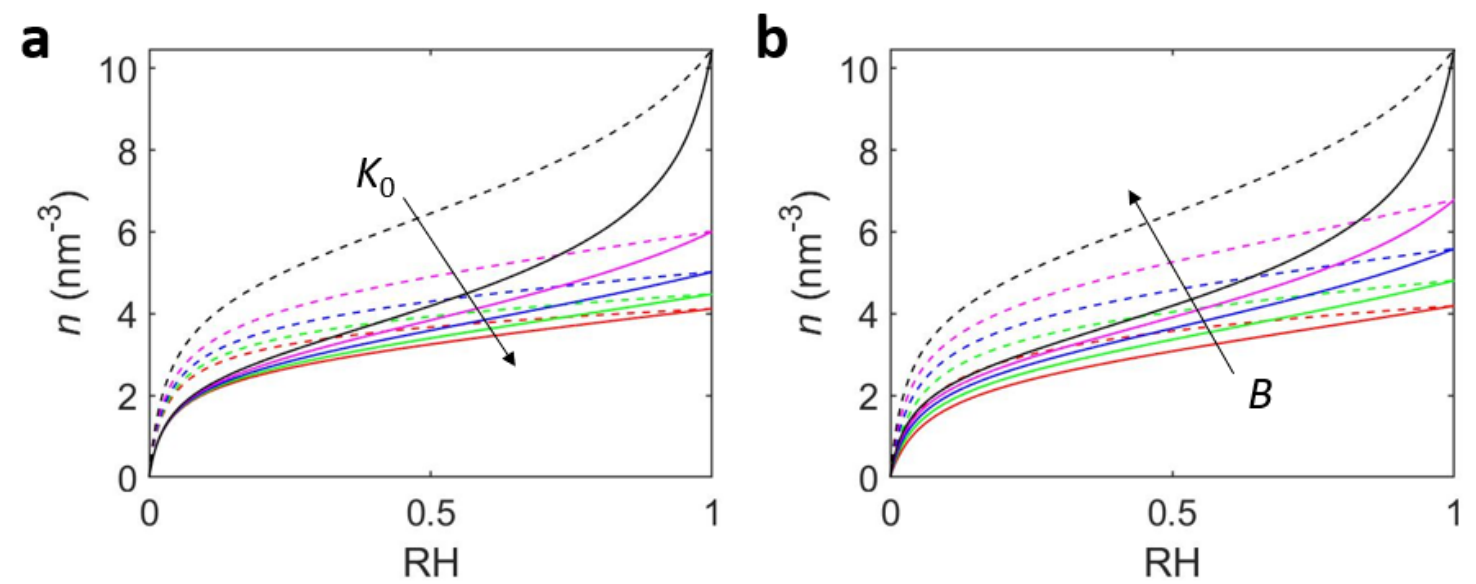

Figure 14 Sorption isotherms under different (a) stiffness $K_{0}$ (b) sorption parameter $B$. The black arrow indicates the direction of increasing $K_{0}$ or $n_{0}$. $K_{0}$ increase from $4 \mathrm{GPa}$ to $5 \mathrm{GPa}$ by a step of $0.25 \mathrm{GPa}$ and are presented by black, magenta, blue, green and red lines, respectively. $B$ increases from $0.005 \mathrm{~Pa}^{-1}$ to $0.01 \mathrm{~Pa}^{-1}$ by a step of $0.00125 \mathrm{~Pa}^{-1}$ and are presented by red, green, blue, magenta and black lines, respectively. The solid and dashed line represent adsorption and desorption isotherms respectively.

Figure 14(b) shows that the sorption isotherm changes from type I to type II and that the hysteresis loop becomes larger upon increasing $B$. As $B$ increases, the stress induced by sorption, which is described by the second term on R.H.S. of Eq.(5), increases. Consequently, the sorption-induced strain (swelling) gets larger. Following a similar reasoning as for describing the influence of $K_{0}$, a higher value of $B$ corresponds to a larger initially closed pores becoming opened during adsorption, which finally contribute to more sorption hysteresis.

\section{Conclusion}

In this paper, we developed a theoretical model combining Dependent Domain Model (DDM) and poromechanics to describe the hysteretic sorption behavior in nanoporous polymers based on the 
mechanisms as revealed by atom-scale molecular simulations. The material is assumed to be composed of a set of elements with different pore sizes. Elements are made 'dependent' by taking into account the mechanical interaction between neighboring elements via a poromechanics framework. The hysteresis of sorption and deformation is modeled by a switch in state (open/close) of elements dependent on strain, based on the mechanism revealed in molecular simulation.

We find that the developed model is capable of modeling sorption hysteresis of nanoporous polymers and captures the coupling mechanism of sorption hysteresis and deformation. Both Finite Element (FE) solution and analytical solution agree with the molecular simulations and experiments. The model solved by FE allows to illustrate that water filling of the porous material follows different distributions upon adsorption and desorption. We further explore how the material properties relate to the sorption hysteresis. A relatively general conclusion can be drawn that a system with softer solid and stronger adsorbent-adsorbate interaction tends to have larger sorption hysteresis for this kind of material.

There have been significant recent advances in modeling sorption hysteresis and sorption-induced deformation of nanoporous materials. While adsorption and deformation phenomena are often treated as separate steps, they are strongly coupled in soft nanoporous media such as intrinsically microporous polymers including cellulose-based materials. In this paper, we combine the framework of poromechanics and the Dependent Domain Theory to capture the coupling between adsorption and deformation in these complex, heterogeneous materials. The developed model is based on the coupling mechanisms found by molecular simulations, thus most of the parameters employed in the model have clear physical meanings. This gives the model a huge advantage in studying the coupling physics of nanoporous polymers in two main aspects. First, this model relates sorption hysteresis explicitly to material properties, which allows us to explain different sorption behaviors for different material systems. Moreover, this model paves the way for the design of materials with tailored desired hysteretic behavior. Second, this model, successfully includes the microscopic coupling mechanism into the macroscopic description. In other words, it can serve as an upscaling model bridging the microscopic mechanism and the macroscopic description. In brief, two concepts - the sorption-deformation coupling and multi-scale modeling - are newly introduced into the domain theory. We believe this model is not only limited to a better description of nanoporous materials but also points to a new direction in modeling sorption hysteresis.

\section{Acknowledgements}


The authors acknowledge the support of the Swiss National Science Foundation (SNSF) (No.143601).

\section{Reference}

(1) Toomey, R.; Freidank, D.; Rühe, J. Swelling Behavior of Thin, Surface-Attached Polymer Networks. Macromolecules 2004, 37 (3), 882-887. https://doi.org/10.1021/ma034737v.

(2) Guyer, R. A.; McCall, K. R. Capillary Condensation, Invasion Percolation, Hysteresis, and Discrete Memory. Phys. Rev. B 1996, 54 (1), 18-21.

https://doi.org/10.1103/PhysRevB.54.18.

(3) Morishige, K.; Shikimi, M. Adsorption Hysteresis and Pore Critical Temperature in a Single Cylindrical Pore. J. Chem. Phys. 1998, 108 (18), 7821-7824.

https://doi.org/10.1063/1.476218.

(4) Burgess, C. G. V.; Everett, D. H.; Nuttall, S. Adsorption Hysteresis in Porous Materials. Pure Appl. Chem. 1989, 61 (11), 1845-1852. https://doi.org/10.1351/pac198961111845.

(5) Neimark, A. V.; Ravikovitch, P. I.; Vishnyakov, A. Adsorption Hysteresis in Nanopores. Phys. Rev. E 2000, 62 (2), R1493-R1496. https://doi.org/10.1103/PhysRevE.62.R1493.

(6) Shi, J.; Avramidis, S. Water Sorption Hysteresis in Wood: I Review and Experimental Patterns - Geometric Characteristics of Scanning Curves. Holzforschung 2017, 71 (4), 307-316. https://doi.org/10.1515/hf-2016-0120.

(7) Urquhart, A. R.; Williams, A. M. Ii.-THE ABSORPTION AND DESORPTION OF WATER BY SODA-BOILED COTTON AT $25^{\circ}$ C. J. Text. Inst. Trans. 1924, 15 (9), T433-T442. https://doi.org/10.1080/19447022408661314.

(8) Smith, S. E. The Sorption of Water Vapor by High Polymers. J. Am. Chem. Soc. 1947, 69 (3), 646-651. https://doi.org/10.1021/ja01195a053.

(9) Filby, E.; Maass, O. The Volume Relations of the System Cellulose and Water. Can. J. Res. 1932, 7 (2), 162-177. https://doi.org/10.1139/cjr32-068.

(10) Urquhart, A. R.; Eckersall, N. The Moisture Relations of Cotton. Vii-A Study of Hysteresis. J. Text. Inst. Trans. 1930, 21 (10), T499-T510.

https://doi.org/10.1080/19447023008661537. 
(11) Sing, K. S. W. Reporting Physisorption Data for Gas/Solid Systems with Special Reference to the Determination of Surface Area and Porosity (Recommendations 1984). Pure Appl. Chem. 1985, 57 (4), 603-619. https://doi.org/10.1351/pac198557040603.

(12) Cohan, L. H. Sorption Hysteresis and the Vapor Pressure of Concave Surfaces. J. Am. Chem. Soc. 1938, 60 (2), 433-435. https://doi.org/10.1021/ja01269a058.

(13) Horikawa, T.; Do, D. D.; Nicholson, D. Capillary Condensation of Adsorbates in Porous Materials. Adv. Colloid Interface Sci. 2011, 169 (1), 40-58. https://doi.org/10.1016/j.cis.2011.08.003.

(14) Chen, M.; Coasne, B.; Guyer, R.; Derome, D.; Carmeliet, J. Role of Hydrogen Bonding in Hysteresis Observed in Sorption-Induced Swelling of Soft Nanoporous Polymers. Nat.

Commun. 2018, 9 (1), 3507. https://doi.org/10.1038/s41467-018-05897-9.

(15) Kulasinski, K.; Guyer, R.; Keten, S.; Derome, D.; Carmeliet, J. Impact of Moisture Adsorption on Structure and Physical Properties of Amorphous Biopolymers.

Macromolecules 2015, 48 (8), 2793-2800. https://doi.org/10.1021/acs.macromol.5b00248.

(16) Coasne, B.; Gubbins, K. E.; Pellenq, R. J.-M. Temperature Effect on Adsorption/Desorption Isotherms for a Simple Fluid Confined within Various Nanopores. Adsorption 2005, 11 (S1), 289-294. https://doi.org/10.1007/s10450-005-5939-y.

(17) Schlaich, A.; Coasne, B. Dispersion Truncation Affects the Phase Behavior of Bulk and Confined Fluids: Coexistence, Adsorption, and Criticality. J. Chem. Phys. 2019, 150 (15), 154104. https://doi.org/10.1063/1.5085431.

(18) Bažant, Z. P.; Bazant, M. Z. Theory of Sorption Hysteresis in Nanoporous Solids: Part I. J. Mech. Phys. Solids 2012, 60 (9), 1644-1659. https://doi.org/10.1016/j.jmps.2012.04.014.

(19) Bazant, M. Z.; Bažant, Z. P. Theory of Sorption Hysteresis in Nanoporous Solids: Part II Molecular Condensation. J. Mech. Phys. Solids 2012, 60 (9), 1660-1675. https://doi.org/10.1016/j.jmps.2012.04.015.

(20) Urquhart, A. R.; Williams, A. M. Ii.-THE ABSORPTION AND DESORPTION OF WATER BY SODA-BOILED COTTON AT $25^{\circ}$ C. J. Text. Inst. Trans. 1924, 15 (9), T433-T442. https://doi.org/10.1080/19447022408661314. 
(21) Chirkova, J.; Andersons, B.; Andersone, I. Study of the Structure of Wood-Related Biopolymers by Sorption Methods. BioResources 2009, 4 (3), 1044-1057.

(22) Preisach, F. Über Die Magnetische Nachwirkung. Zeitschrift für Phys. 1935, 94 (5-6), 277-302. https://doi.org/10.1007/BF01349418.

(23) Everett, D. H.; Whitton, W. I. A General Approach to Hysteresis. Trans. Faraday Soc. 1952, 48 (May), 749. https://doi.org/10.1039/tf9524800749.

(24) Everett, D. H.; Smith, F. W. A General Approach to Hysteresis. Part 2: Development of the Domain Theory. Trans. Faraday Soc. 1954, 50, 187. https://doi.org/10.1039/tf9545000187.

(25) Coasne, B.; Gubbins, K. E.; Pellenq, R. J.-M. Domain Theory for Capillary Condensation Hysteresis. Phys. Rev. B 2005, 72 (2), 024304. https://doi.org/10.1103/PhysRevB.72.024304.

(26) Derluyn, H.; Derome, D.; Carmeliet, J.; Stora, E.; Barbarulo, R. Hysteretic Moisture Behavior of Concrete: Modeling and Analysis. Cem. Concr. Res. 2012, 42 (10), 13791388. https://doi.org/10.1016/j.cemconres.2012.06.010.

(27) Pinson, M. B.; Zhou, T.; Jennings, H. M.; Bazant, M. Z. Inferring Pore Connectivity from Sorption Hysteresis in Multiscale Porous Media. J. Colloid Interface Sci. 2018, 532, 118 127. https://doi.org/10.1016/j.jcis.2018.07.095.

(28) PERALTA, P. Modeling Wood Moisture Sorption Hysteresis Using the IndependentDomain Theory. Wood Fiber Sci. 1995, 27 (3), 250-257.

(29) Peralta, P. N.; Bangi, A. P. Modeling Wood Moisture Sorption Hysteresis Based on Similarity Hypothesis. Part 2. Capillary_Radii Approach. Wood Fiber Sci. 1998, 30 (2), $148-154$.

(30) Patera, A.; Derluyn, H.; Derome, D.; Carmeliet, J. Influence of Sorption Hysteresis on Moisture Transport in Wood. Wood Sci. Technol. 2016, 50 (2), 259-283. https://doi.org/10.1007/s00226-015-0786-9.

(31) Shi, J.; Avramidis, S. Water Sorption Hysteresis in Wood: II Mathematical Modeling Functions beyond Data Fitting. Holzforschung 2017, 71 (4), 317-326. https://doi.org/10.1515/hf-2016-0121. 
(32) Guyer, R. a.; Kim, H. A.; Derome, D.; Carmeliet, J.; TenCate, J. Hysteresis in Modeling of Poroelastic Systems: Quasistatic Equilibrium. Phys. Rev. E 2011, 83 (6), 061408. https://doi.org/10.1103/PhysRevE.83.061408.

(33) Coussy, O. Poromechanics; John Wiley \& Sons, Ltd: Chichester, UK, 2003. https://doi.org/10.1002/0470092718.

(34) Gor, G. Y.; Huber, P.; Weissmüller, J. Elastocapillarity in Nanopores: Sorption Strain from the Actions of Surface Tension and Surface Stress. Phys. Rev. Mater. 2018, 2 (8), 086002. https://doi.org/10.1103/PhysRevMaterials.2.086002.

(35) Vandamme, M. Coupling between Adsorption and Mechanics (and Vice Versa). Curr. Opin. Chem. Eng. 2019, 24, 12-18. https://doi.org/10.1016/j.coche.2018.12.005.

(36) Brochard, L.; Vandamme, M.; Pellenq, R. J.-M. Poromechanics of Microporous Media. J. Mech. Phys. Solids 2012, 60 (4), 606-622. https://doi.org/10.1016/j.jmps.2012.01.001.

(37) Coasne, B.; Weigel, C.; Polian, A.; Kint, M.; Rouquette, J.; Haines, J.; Foret, M.; Vacher, R.; Rufflé, B. Poroelastic Theory Applied to the Adsorption-Induced Deformation of Vitreous Silica. J. Phys. Chem. B 2014, 118 (49), 14519-14525.

https://doi.org/10.1021/jp5094383.

(38) Zhang, Y. Mechanics of Adsorption-Deformation Coupling in Porous Media. J. Mech. Phys. Solids 2018, 114, 31-54. https://doi.org/10.1016/j.jmps.2018.02.009.

(39) Kulasinski, K.; Guyer, R.; Derome, D.; Carmeliet, J. Poroelastic Model for AdsorptionInduced Deformation of Biopolymers Obtained from Molecular Simulations. Phys. Rev. E 2015, 92 (2), 022605. https://doi.org/10.1103/PhysRevE.92.022605.

(40) Carmeliet, J.; Derome, D.; Dressler, M.; Guyer, R. A. Nonlinear Poro-Elastic Model for Unsaturated Porous Solids. J. Appl. Mech. 2013, 80 (2), 020909. https://doi.org/10.1115/1.4007921.

(41) Pinson, M. B.; Zhou, T.; Jennings, H. M.; Bazant, M. Z. Inferring Pore Connectivity from Sorption Hysteresis in Multiscale Porous Media. J. Colloid Interface Sci. 2018, 532 (July 2015), 118-127. https://doi.org/10.1016/j.jcis.2018.07.095.

(42) Zhou, T.; Ioannidou, K.; Ulm, F.-J.; Bazant, M. Z.; Pellenq, R. J. M. Multiscale Poromechanics of Wet Cement Paste. Proc. Natl. Acad. Sci. 2019, 116 (22), $10652-$ 10657. https://doi.org/10.1073/pnas.1901160116. 
(43) Chen, M.; Coasne, B.; Derome, D.; Carmeliet, J. Coupling of Sorption and Deformation in Soft Nanoporous Polymers: Molecular Simulation and Poromechanics. J. Mech. Phys. Solids 2020, 137, 103830. https://doi.org/10.1016/j.jmps.2019.103830.

(44) Zhou, T.; Ioannidou, K.; Masoero, E.; Mirzadeh, M.; Pellenq, R. J. M.; Bazant, M. Z. Capillary Stress and Structural Relaxation in Moist Granular Materials. Langmuir 2019, 35 (12), 4397-4402. https://doi.org/10.1021/acs.langmuir.8b03400.

(45) Budkov, Y. A.; Kolesnikov, A. L.; Georgi, N.; Kiselev, M. G. A Statistical Theory of Cosolvent-Induced Coil-Globule Transitions in Dilute Polymer Solution. J. Chem. Phys. 2014, 141 (1), 014902. https://doi.org/10.1063/1.4884958.

(46) Budkov, Y. A.; Kolesnikov, A. L. Statistical Description of Co-Nonsolvency Suppression at High Pressures. Soft Matter 2017, 13 (45), 8362-8367. https://doi.org/10.1039/c7sm01637a.

(47) Evans, J. D.; Krause, S.; Kaskel, S.; Sweatman, M. B.; Sarkisov, L. Exploring the Thermodynamic Criteria for Responsive Adsorption Processes. Chem. Sci. 2019, 10 (19), 5011-5017. https://doi.org/10.1039/C9SC01299K.

(48) Krause, S.; Bon, V.; Senkovska, I.; Stoeck, U.; Wallacher, D.; Többens, D. M.; Zander, S.; Pillai, R. S.; Maurin, G.; Coudert, F.-X.; Kaskel, S. A Pressure-Amplifying Framework Material with Negative Gas Adsorption Transitions. Nature 2016, 532 (7599), 348-352. https://doi.org/10.1038/nature17430.

(49) Mihranyan, A.; Llagostera, A. P.; Karmhag, R.; Strømme, M.; Ek, R. Moisture Sorption by Cellulose Powders of Varying Crystallinity. Int. J. Pharm. 2004, 269 (2), 433-442. https://doi.org/10.1016/j.ijpharm.2003.09.030.

(50) Chen, M.; Coasne, B.; Guyer, R.; Derome, D.; Carmeliet, J. Molecular Simulation of Sorption-Induced Deformation in Atomistic Nanoporous Materials. Langmuir 2019, 35 (24), 7751-7758. https://doi.org/10.1021/acs.langmuir.9b00859.

(51) Coasne, B. Multiscale Adsorption and Transport in Hierarchical Porous Materials. New J. Chem. 2016, 40 (5), 4078-4094. https://doi.org/10.1039/C5NJ03194J.

(52) Jeromenok, J.; Weber, J. Restricted Access: On the Nature of Adsorption/Desorption Hysteresis in Amorphous, Microporous Polymeric Materials. Langmuir 2013, 29 (42), 12982-12989. https://doi.org/10.1021/la402630s. 
(53) Heuchel, M.; Fritsch, D.; Budd, P. M.; McKeown, N. B.; Hofmann, D. Atomistic Packing Model and Free Volume Distribution of a Polymer with Intrinsic Microporosity (PIM-1). J. Memb. Sci. 2008, 318 (1-2), 84-99. https://doi.org/10.1016/j.memsci.2008.02.038.

(54) Gor, G. Y.; Bernstein, N. Revisiting Bangham's Law of Adsorption-Induced Deformation: Changes of Surface Energy and Surface Stress. Phys. Chem. Chem. Phys. 2016, 18 (14), 9788-9798. https://doi.org/10.1039/C6CP00051G.

(55) Kolesnikov, A. L.; Georgi, N.; Budkov, Y. A.; Möllmer, J.; Hofmann, J.; Adolphs, J.; Gläser, R. Effects of Enhanced Flexibility and Pore Size Distribution on AdsorptionInduced Deformation of Mesoporous Materials. Langmuir 2018, 34 (25), 7575-7584. https://doi.org/10.1021/acs.langmuir.8b00591.

(56) Balzer, C.; Cimino, R. T.; Gor, G. Y.; Neimark, A. V.; Reichenauer, G. Deformation of Microporous Carbons during N 2 , Ar, and CO 2 Adsorption: Insight from the Density Functional Theory. Langmuir 2016, 32 (32), 8265-8274.

https://doi.org/10.1021/acs.langmuir.6b02036.

(57) Kowalczyk, P.; Balzer, C.; Reichenauer, G.; Terzyk, A. P.; Gauden, P. A.; Neimark, A. V. Using In-Situ Adsorption Dilatometry for Assessment of Micropore Size Distribution in Monolithic Carbons. Carbon N. Y. 2016, 103, 263-272. https://doi.org/10.1016/j.carbon.2016.02.080.

(58) Ravikovitch, P. I.; Neimark, A. V. Density Functional Theory Model of Adsorption Deformation. Langmuir 2006, 22 (26), 10864-10868. https://doi.org/10.1021/la061092u.

(59) Hill, R. The Elastic Behaviour of a Crystalline Aggregate. Proc. Phys. Soc. Sect. A 1952, 65 (5), 349-354. https://doi.org/10.1088/0370-1298/65/5/307. 


\section{Appendix}

\section{Mesh dependent study}

We test three different degrees of discretization: $20 \times 20,30 \times 30$ and $40 \times 40$. The comparisons of the PSD and sorption isotherms are given in Figure A1. It shows that the results of the three cases are almost the same. Quantitatively, the relative difference between sorption amount of the case $20 \times 20$ and $40 \times 40$ is $4.34 \%$. Thus, we use the model of $20 \times 20$ for further analysis in the main text for efficiency without much loss of accuracy.
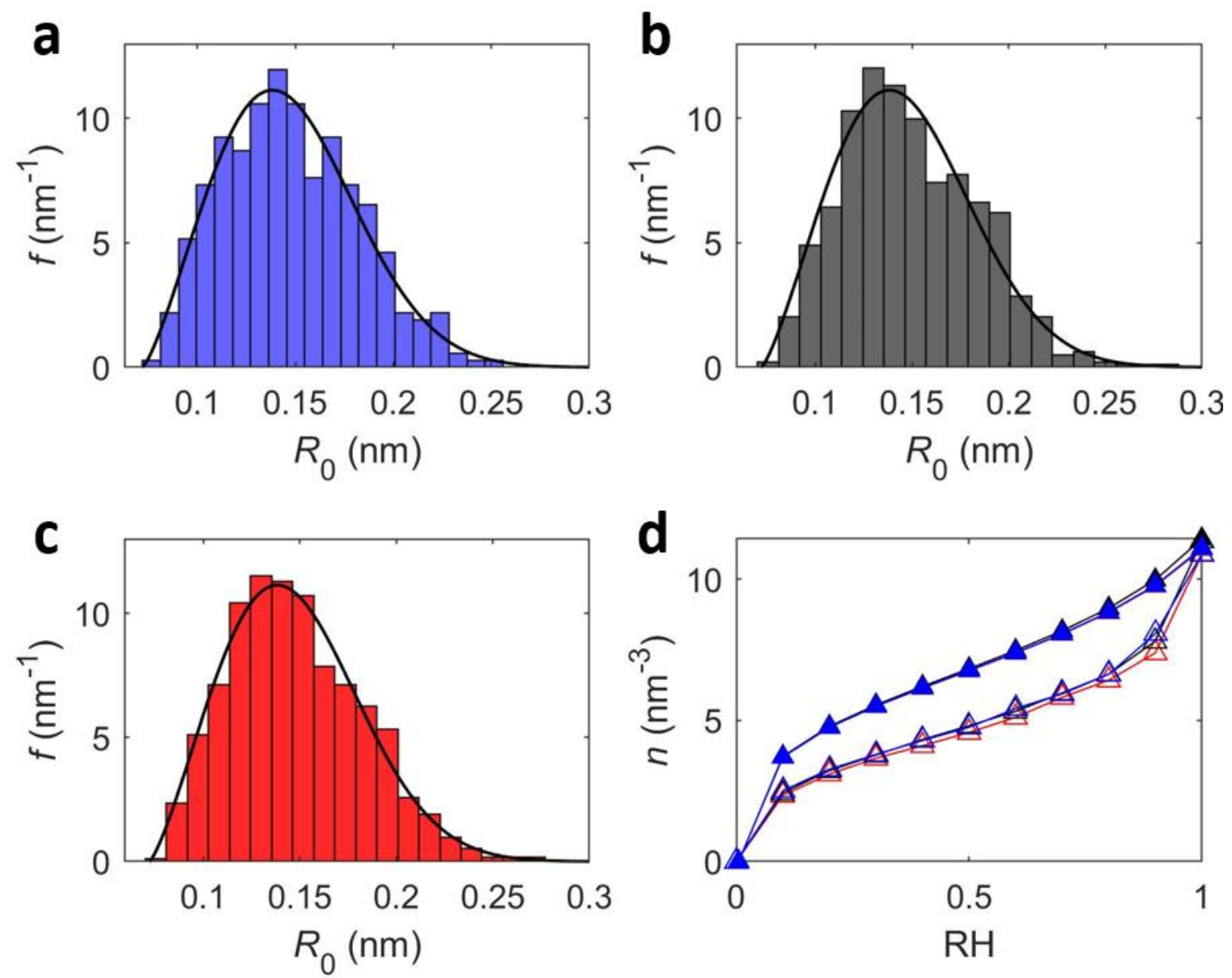

Figure A1 Pore size distribution generated based on the determined Weibull distribution in FE model with a degree of discretization of (a) $20 \times 20$; (b) $30 \times 30$ and (c) $40 \times 40$. The sorption isotherms based on the three models are compared in (d). The blue, black and red triangles represent the case of $20 \times 20,30 \times 30$ and $40 \times 40$ respectively. The open and closed triangles represent adsorption and desorption respectively. 